\title{
Hot Gas In and Around Elliptical Galaxies
}

\author{
William G. Mathews ${ }^{1}$ and Fabrizio Brighenti ${ }^{1,2}$ \\ ${ }^{1}$ University of California Observatories/Lick Observatory, Department of Astronomy and \\ Astrophysics, University of California, Santa Cruz, CA 95064 mathews@ucolick.org \\ ${ }^{2}$ Dipartimento di Astronomia, Università di Bologna, via Ranzani 1, Bologna 40127, Italy \\ brighenti@bo.astro.it
}

KEYWORDS: elliptical galaxies, X-rays, cooling flows, galaxy groups, galaxy clusters

\begin{abstract}
We review the origin, evolution and physical nature of hot gas in elliptical galaxies and associated galaxy groups. Unanticipated recent X-ray observations with Chandra and XMM indicate much less cooling than previously expected. Consequently, many long-held assumptions need to be reexamined or discarded and new approaches must be explored. Chief among these are the role of heating by active galactic nuclei, the influence of radio lobes on the hot gas, details of the cooling process, possible relation between the hot and colder gas in elliptical galaxies, and the complexities of stellar enrichment of the hot gas.
\end{abstract}

\begin{abstract}
CONTENTS

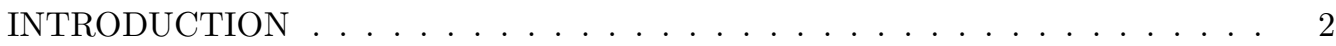

OVERVIEW OF HOT GAS FLOWS IN AND NEAR ELLIPTICAL GALAXIES . 3

BASIC PROPERTIES OF ELLIPTICAL GALAXIES AND THEIR HOT GAS . . 5

HYDROSTATIC EQUILIBRIUM AND MASS DETERMINATIONS . . . . . . . . 8

GAS DYNAMICAL EQUATIONS AND CLASSIC COOLING FLOWS . . . . . 15

SIMPLE COOLING FLOW MODELS . . . . . . . . . . . . . . . . . . . . 18

THE COOLING PARADOX - DOES THE GAS COOL? . . . . . . . . . . . . . . 24

Spectroscopic and Morphological Cooling Rates . . . . . . . . . . . . . . . . 24

Warm and Cold Gas at $T<10^{5} K \ldots \ldots \ldots \ldots \ldots$

Dust in Elliptical Galaxies . . . . . . . . . . . . . . . . . . . . . . 26

Origin of Warm Gas, Cold Gas and Dust . . . . . . . . . . . . . . . . . . . 27

Hiding the Cooling Gas . . . . . . . . . . . . . . . . . . 28

COOLING FLOWS HEATED BY ACTIVE NUCLEI . . . . . . . . . . . . . . . 29

Chandra Observations and Entropy Floors . . . . . . . . . . . . . . . . . . . . . 29

AGN Heating . . . . . . . . . . . . . . . . . . . . . . . . . . . . . 31

Cooling Flow Models with Heating . . . . . . . . . . . . . . . . . . . . . . . . . . 31

Models of X-ray Cavities . . . . . . . . . . . . . . . . . . . . . . . 34

ROTATION AND MAGNETIC FIELDS . . . . . . . . . . . . . . 35

ABUNDANCES IN THE HOT GAS . . . . . . . . . . . . . . . . . . 35
\end{abstract}




\section{INTRODUCTION}

Recent Chandra and XMM X-ray observations of the hot gas in elliptical galaxies and galaxy clusters have radically upset the traditional concept of cooling flows. On the other hand, these same observations may reveal new ways of understanding the origin and evolution of this gas. The most unexpected new discovery has been the absence in XMM spectra of emission from gas at intermediate or low temperatures, implying that the cooling gas is somehow hidden from view or that the cooling rate is much less than previously thought. Chandra X-ray images show that the hot gas deep inside $\mathrm{E}$ and $\mathrm{cD}$ galaxies near the centers of cooling flows is often highly disturbed. Evidently, massive black holes, thought to inhabit the cores of all stellar bulges, may become energy sources when stimulated by inflowing hot gas.

Can the central heating visible in Chandra images explain the absence of cooling in XMM spectra? This is one of many fundamental cooling flow questions that we review here. There is also a problem with terminology. Since the spectral evidence for cooling has weakened, the "cooling flow" concept may no longer be appropriate. Nevertheless, in keeping with accepted usage we occasionally use "cooling flow" when "galactic flow" might be preferred. In any case, the hot gas in and around elliptical galaxies is of particular astronomical interest because its origin and metal enrichment are closely entwined with the merger origin of central elliptical (E) galaxies in galaxy groups. A better understanding of the gas is likely to clarify baryonic structure formation in general. Fortunately, even with our currently incomplete understanding of its origin and evolution, the hot gas can contribute important information about the stellar kinematics and mass to light ratios in elliptical galaxies.

This review is necessarily limited to elliptical galaxies of moderate or high luminosity. X-ray emission from hot gas in low luminosity E galaxies is difficult to observe since it is masked by X-rays from stellar sources. We also emphasize whenever possible those X-ray ellipticals that are relatively undisturbed by recent mergers, stripping interactions with hot cluster gas or powerful radio sources. Although the X-ray properties of S0 galaxies can resemble those of E galaxies, we restrict our discussion to E galaxies owing to the uniformity of their optical structures and their less controversial origin.

The evolution and physical condition of the hot gas in rich clusters of galaxies and in individual E galaxies are similar in many ways. But there are some important distinctions. Because smaller structures are often older in our hierarchical universe, the hot gas in elliptical galaxies and their associated galaxy groups may on average be less disturbed by ongoing mergers than their younger, more massive cluster counterparts. X-ray bright $\mathrm{E}$ galaxies that are rather isolated and, one hopes, undisturbed may contain in their metallicity gradient unique information about star and galaxy formation in the distant past. This vital information is lost when such groups merge and mix to form rich clusters. Another key attribute of hot gas on galactic scales is the possible importance of stellar mass loss and continued Type Ia supernova activity in supplying and enriching the hot gas. Finally, the properties of the hot gas can be determined with more confidence on galactic scales where the gravitational potential is better known from the stellar light.

Notwithstanding their astrophysical importance, proximity and relative ease of interpretation, far less Chandra and XMM data are available for E galaxies and 
their groups than for galaxy clusters. Both telescopes were launched in 1999. As we write this review in the Fall of 2002 no XMM spectrum of a bright, relatively isolated, group-centered elliptical galaxy has appeared in the standard journals, but we expect this data to appear very soon.

Our review is not intended to be a comprehensive review of all the literature that has appeared since the excellent previous cooling flow reviews by Sarazin (1986), Fabbiano (1989), Sarazin (1990) and Fabian (1994). This is due in part to the rapid increase in the quality of the observations which tend to eclipse previous studies only a few years older. Our primary emphasis is to compare observations with theoretical expectations and to bring attention to the frequent dissonance between the two. This approach is complementary to the recent ARAA review of the X-ray properties of galaxy groups by Mulchaey (2000) who presents a more complete discussion of the observations.

\section{OVERVIEW OF HOT GAS FLOWS IN AND NEAR ELLIP- TICAL GALAXIES}

Optical evidence indicates that many or most massive elliptical galaxies formed at high redshifts $(z \gtrsim 2)$ and there is strong theoretical evidence that they formed by mergers in group environments. Later, many ellipticals and their groups merged into massive galaxy clusters. Those that did not, the so-called isolated ellipticals, are surrounded by unmerged group companions and hot gas. As a consequence of their formation and galactic dynamics, big elliptical galaxies are often found near the centers of groups and rich clusters. It is natural that the diffuse thermal gas contained in these hot stellar systems is also hot.

There are two main sources of hot gas in elliptical galaxies: internal and external. Evolving stars inside the elliptical galaxy continuously eject gas at a rate $\sim 1.3\left[L_{B} /\left(10^{11} L_{B, \odot}\right)\right] M_{\odot} \mathrm{yr}^{-1}$. It is generally assumed that gas ejected by orbiting red giant stars passes through shocks and is raised to the stellar kinematic temperature $T_{*} \approx T_{\text {vir }} \approx \mu m_{p} \sigma^{2} / k \sim 10^{7} \mathrm{~K} \sim 1 \mathrm{keV}$ where $\mu$ is the molecular weight and $\sigma$ is the stellar velocity dispersion. Type Ia supernovae provide some additional heating. The large X-ray luminosities of massive E galaxies, $L_{x} \sim 10^{40}-10^{43} \mathrm{ergs} \mathrm{s}^{-1}$ for $L_{B}>3 \times 10^{10} L_{B, \odot}$, indicate that most of the internally produced gas is currently trapped in the galactic or group potential. But at early times, when most of the galactic stars were forming, Type II supernovae were frequent enough to drive winds of enriched gas into the local environment. Gas expelled in this manner from both central and non-central group or cluster galaxies has enriched the hot gas far beyond the stellar image of the central luminous $\mathrm{E}$ or $\mathrm{cD}$ galaxy. In time, some of this local (circumgalactic) gas flows back into the central galaxy, providing an external source of gas. Continued accretion from the ambient cosmological flow that is gravitationally bound to the group or cluster is an additional source of external gas. As diffuse external gas arrives after having fallen through the deeper potential well of the surrounding group/cluster, it is shock-heated to the virial temperature of the galaxy group/cluster. This more distant accreted and shocked gas is hotter than gas virialized to $T_{*}$ deeper in the stellar potential of the E galaxy, and the two together form an an outwardly increasing gas temperature that is commonly observed. The accumulated mass of circumgalactic gas with $T>T_{*}$, bound to the dark matter halo, can extend far beyond the optical image of the luminous $\mathrm{E}$ galaxy. 
The electron density of the hot gas in giant elliptical galaxies is typically $n(0) \sim$ $0.1 \mathrm{~cm}^{-3}$ at the center and declines with radius as $n \propto r^{-1.25 \pm 0.25}$. Depending on its spatial extent, the total mass of hot gas in massive E galaxies varies up to at least several $10^{10} M_{\odot}$ or about $\lesssim 1$ percent of the total stellar mass. This is only a few times less than the gas to stellar mass ratio in the Milky Way. The iron abundance in the hot gas in ellipticals increases from $z_{F e} \sim 0.2-0.4$ beyond the optical image to $z_{F e} \sim 1-2$ in the center where it is evidently enriched by Type Ia supernovae.

To a good approximation, the hot gas in and near elliptical galaxies is in hydrostatic equilibrium. Supersonic winds are not common in well-observed massive ellipticals $\left(L_{B} \gtrsim 3 \times 10^{10} L_{B, \odot}\right)$ because they would have low gas densities and much lower X-ray luminosities. A characteristic feature of the hot gas is that the the dynamical and sound crossing times are nearly equal, as expected in hydrostatic equilibrium, and both are much less than the radiative cooling time. Any cooling-induced flow is therefore highly subsonic, essentially in hydrostatic equilibrium. This equilibrium can be disturbed by mergers or by energy released in an active galactic nucleus (AGN) associated with a supermassive black hole in the core of the central elliptical. Nevertheless, by assuming hydrostatic equilibrium, the total mass distribution $M_{t o t}(r)$ has been determined for many galaxies and clusters from X-ray observations. If a galaxy group has been relatively undisturbed for many Gyrs, the metal enrichment in the hot gas may retain a memory of the (largely SNII-driven) galactic winds that occurred in the distant past. We are just beginning to exploit this gold mine of information.

In addition to the accretion shock, hot gas in ellipticals is heated further by Type Ia supernovae and by dissipation of mass lost from orbiting stars. As a consequence of the $\sim 0.7 M_{\odot}$ of iron that each Type Ia is thought to contribute, the observed iron abundance in the hot gas is a measure of the supernovae heating for a given flow model. In low luminosity ellipticals, which have shallower gravitational potentials, current supernova heating may be sufficient to drive a galactic outflow. But in optically bright, X-ray luminous E galaxies, supernova heating is generally unable to balance the radiative losses from the hot plasma that produces the X-ray emission we observe. Paradoxically, the loss of radiative energy from the hot gas does not result in a proportional decrease in the local gas temperature. Instead, this loss of thermal energy is immediately compensated by $P d v$ compression in the gravitational potential of the galaxy/group that maintains the temperature $\sim T_{\text {vir }}$ necessary to support the surrounding atmosphere of hot gas. However, near the center of the flow where there is no deeper galactic potential, catastrophic radiative cooling could in principle occur.

But something is wrong, perhaps radically wrong, with this simple "cooling flow" model. An estimate of the mass cooling rate required to generate the observed X-ray luminosity in a bright E galaxy, $\dot{M} \approx\left(2 \mu m_{p} / 5 k T\right) L_{x, b o l}$ reveals that several $10^{10} M_{\odot}$ of gas should have cooled somewhere within the galaxy over a Hubble time. The cooling cannot be too concentrated because this mass exceeds the masses of known central black holes by factors of 10 - 20 and its gravitational attraction on the hot gas would produce an unobserved central peak in X-ray emission. The traditional solution to this problem is to invoke an ad hoc mass "dropout" assumption in which the gas somehow cools throughout a large volume of the flow. Unfortunately, such distributed cooling cannot result from thermal instabilities following small perturbations in the hot gas. Instead, larger perturbations formed in turbulent regions may be required, but the details 
remain uncertain.

Even more astonishing, detailed X-ray spectra taken with XMM show little or no emission from ions cooling at temperatures much below $\sim T_{v i r} / 2$, implying that little gas completely cools or perhaps none at all! There are two possible explanations: (1) the gas is cooling, but it is not visible and/or (2) the gas is truly not cooling and the radiative losses are offset by some source of heating.

In principle, X-ray emission from cooling gas can be attenuated or hidden (1) by spatially distributed colder gas that absorbs softer X-rays or (2) if the cooling is somehow accelerated so that the $\mathrm{X}$-ray line emission from the cooling gas is reduced. Although X-ray spectra provide some support for distributed absorption at energies $\lesssim 1 \mathrm{keV}$, the absorbing gas would need to be colder, $T_{a b s} \lesssim 10^{6} \mathrm{~K}$, spatially extended and very massive $M_{a b s} \approx 10^{10}(r / 10 \mathrm{kpc})^{2} M_{\odot}$. Emission from this absorbing gas has not been observed. Alternatively, rapid cooling may be possible in localized regions of high metallicity such as remnants of Type Ia explosions or if cold gas rapidly mixes with the hot gas. Cooling may also be very rapid in the dust-rich gas recently ejected from evolving stars. These processes have not been studied in detail.

The currently most popular explanation for the absence of spectroscopic evidence for cooling is that the hot gas is being heated by active galactic nuclei (AGN) in the cores of flow-centered giant ellipticals. This is reasonable since almost all bright $\mathrm{E}$ galaxies have extended non-thermal radio emission at some level in their cores. Additional support for this heating hypothesis is provided by the spectacular X-ray images from Chandra in which the hot gas in the central regions of virtually all elliptical galaxies observed so far appears to be highly disturbed and irregular. In some cases bubbles (X-ray cavities) filled with relativistic or superheated gas have displaced the $1 \mathrm{keV}$ thermal gas and appear to be buoyantly floating upward in the atmosphere. Many observers have noted with astonishment that the gas just adjacent to the bubbles is cooler than average, indicating that the holes have not been recently produced by strong shocks.

Certainly the luminosities of typical AGN are sufficient to offset the X-ray luminosities $L_{x}$ of classic cooling flows and maintain their gas temperature near $T_{v i r}$. However, the problem with the heating hypothesis, which is widely unappreciated, is that it is difficult to communicate this AGN energy to the hot gas at larger radii and still preserve the globally observed hot gas temperature and density profiles.

\section{BASIC PROPERTIES OF ELLIPTICAL GALAXIES AND THEIR HOT GAS}

If elliptical galaxies were perfectly homologous stellar systems with identical stellar populations, then the "central" velocity dispersion $\sigma_{o}$, stellar mass $M$ and half-light (effective) radius $R_{e}$ would be related by the virial theorem, $\sigma_{o}^{2}=$ $\kappa\left(M / R_{e}\right)=\kappa\left(L_{V} / R_{e}\right)\left(M / L_{V}\right)$ with constant $\kappa$. Instead, non-homology and/or stellar population variations conspire to place elliptical galaxies on a nearby fundamental plane $\sigma_{o}^{2} \propto\left(L_{V} / R_{e}\right)\left[R_{e}^{0.22} \sigma_{o}^{0.49}\right]$, implying that $\kappa\left(M / L_{V}\right) \propto R_{e}^{0.22} \sigma_{o}^{0.49} \propto$ $M^{0.24} R_{e}^{-0.02} \propto L_{V}{ }^{0.32} R_{e}^{-0.03}$ (Dressler et al. 1987; Djorgovski \& Davis 1987). The width of the fundamental plane is remarkably small (Renzini \& Ciotti 1993). In projection the fundamental plane indicates that the binding energy per unit mass decreases with stellar (or galactic) mass, $\sigma_{o}^{2} \propto M^{0.32}$ (Faber et al. 1997), i.e., hot 
interstellar gas is less bound in low mass ellipticals. This regularity in the global properties of elliptical galaxies is useful in interpreting the X-ray emission from the hot interstellar gas they contain.

The internal structure is also quite uniform among massive E galaxies. For those with de Vaucouleurs $\left(r^{1 / 4}\right)$ stellar profiles the stellar distribution is completely determined by the optical half-light radius $R_{e}$ and the total stellar mass $M_{* t}$. Provided the dark halos consist of cold, non-interacting particles, the halo density profile is determined by its virial mass, $M_{d h}$, the mass enclosing a cosmic overdensity of $\sim 100$ relative to the critical density $\rho_{c}=3 H_{0}^{2} / 8 \pi G$ in a flat $\Lambda$ CDM universe with $\Omega_{m}=0.3$ (Eke, Navarro, \& Frenk 1998; Bullock et al. 2000). If elliptical galaxies formed by mergers in galaxy groups, as generally believed, then the hot gas they contain must be understood in this evolutionary context. The $r^{1 / 4}$ stellar density profile in ellipticals and the high incidence ( 50 percent) of counter-rotating or kinematically independent stellar cores are natural consequences of hierarchical mergers (Hernquist \& Barnes 1991; Bender 1996). While many massive E galaxies currently reside in the dense cores of rich galaxy clusters, the orbital velocities of cluster member galaxies are too high for efficient mergers.

In spite of these regularities, giant elliptical galaxies come in two flavors depending on their mass or optical luminosity. Low luminosity ellipticals have power law central stellar density profiles, disky isophotes, and oblate symmetry consistent with their moderate rotation. Ellipticals of high luminosity have flatter stellar cores within a break radius $r_{b}$ (typically a few percent of $R_{e}$ ), boxy isophotes, and have aspherical structures caused by anisotropic stellar velocities, not by rotation which is generally small (Kormendy \& Bender 1996; Faber et al. 1997; Lauer et al. 1998). Nevertheless, many of the most luminous E0 and E1 galaxies are thought to be intrinsically spherical to a reasonable approximation (Merritt \& Tremblay 1996) and these galaxies are among the most luminous in X-rays. The transition from power-law to core ellipticals occurs gradually over $-20 \gtrsim M_{V} \gtrsim-22$ where both types coexist on the same fundamental plane. Finally, optical spectra of ellipticals often show evidence of a (sub)population of younger stars, particularly in ellipticals with power-law profiles (e.g. Trager et al. 2000; Terlevich \& Forbes 2002).

The X-ray luminosities $L_{x}$ of elliptical galaxies correlate with $L_{B}$ in a manner that also exhibits a transition with increasing mass (Canizares et al. 1987; Donnelly et al. 1990; White \& Sarazin 1991; Fabbiano, Kim \& Trinchieri 1992; Eskridge, Fabbiano, \& Kim 1995a,b,c; Davis \& White 1996; Brown \& Bregman 1998; Beuing et al. 1999). For elliptical galaxies of low luminosity (i.e. $\left.L_{B} \lesssim L_{B, \text { crit }}=3 \times 10^{9} L_{B, \odot}\right)$ O'Sullivan et al. (2001) find that the bolometric $\mathrm{X}$-ray and optical luminosities are approximately proportional, $L_{x} \propto L_{B}$. The $\mathrm{X}$-ray emission from these galaxies is apparently dominated by low-mass X-ray binary stars with a different (typically harder) spectrum than that of the interstellar gas (Brown \& Bregman 2001). The brightest stellar X-ray sources can be individually resolved with Chandra (e.g. Sarazin, Irwin \& Bregman 2000; Blanton, Sarazin, \& Irwin 2001a). However, the X-ray emission from more luminous ellipticals $\left(L_{B} \gtrsim L_{B, \text { crit }}\right)$ varies approximately as $L_{x} \propto L_{B}^{2}$ (O'Sullivan et al. 2001), clearly indicating a non-stellar origin, i.e., the hot gas. As shown in Figure 1, the scatter about this correlation is enormous, $L_{x}$ varies by almost two orders of magnitude for galaxies with similar $L_{B}$.

The large scatter in the $L_{x} \propto L_{B}^{2}$ correlation has received much attention but 


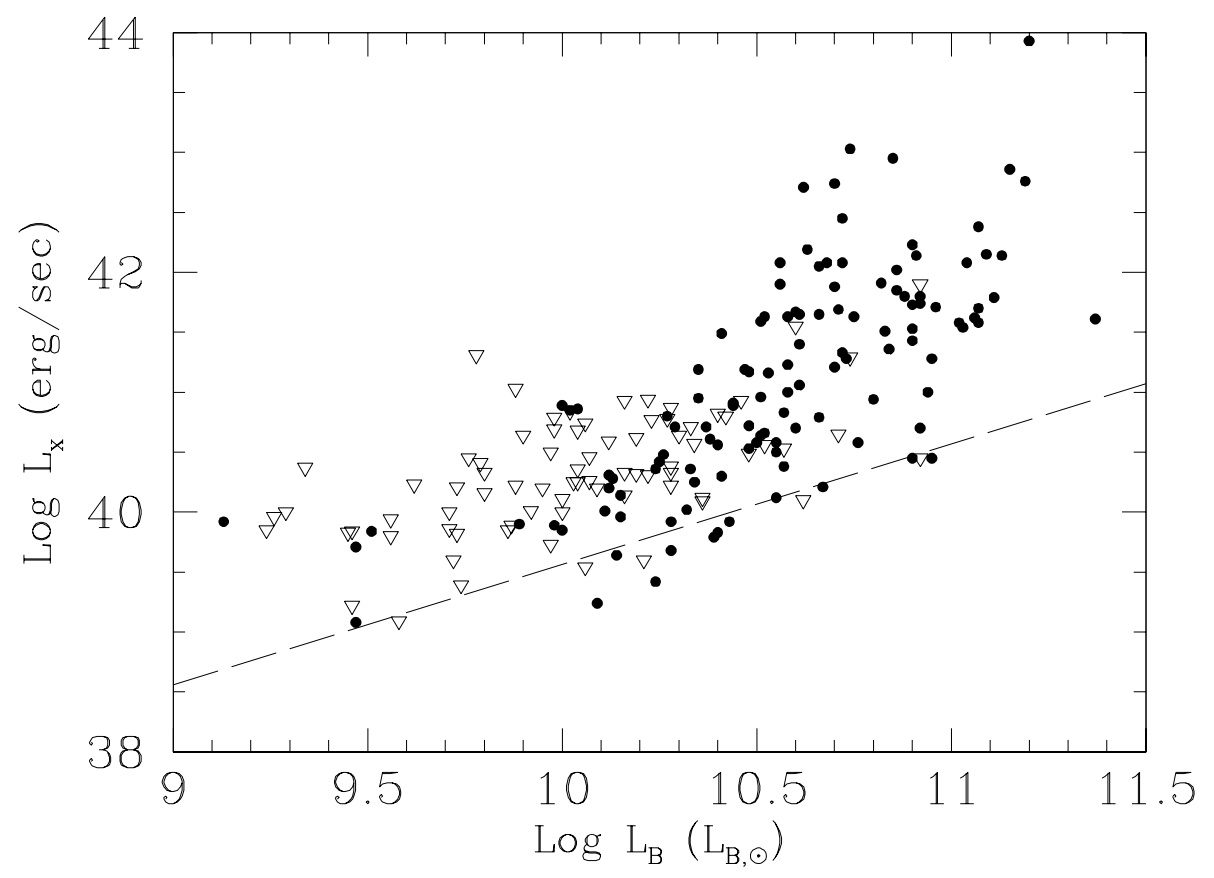

Figure 1: A plot of the bolometric X-ray luminosity and B-band optical luminosity for elliptical galaxies ( $\mathrm{RC} 3$ type $\mathrm{T} \leq-4$ ) from the compilation of O'Sullivan et al. (2001). X-ray detections are shown with filled circles and upper limits with open triangles. The dashed line is an approximate locus of the total luminosity $L_{x, *} \propto L_{B}$ of stellar and other discrete sources also from O'Sullivan et al. 
an explanation in terms of some specific environmental or intrinsic property of the galaxies has been elusive (e.g. Eskridge, Fabbiano, \& Kim 1995a,b,c). White and Sarazin (1991) and Henriksen \& Cousineau (1999) find that ellipticals having other massive galaxies nearby have systematically lower $L_{x} / L_{B}$ while Brown \& Bregman (2000) find a positive correlation with $L_{x} / L_{B}$ and the local density of galaxies. Pellegrini (1999) presented evidence that ellipticals with power law profiles have much smaller range in $L_{x} / L_{B}$, as might be expected if a larger fraction of $L_{x}$ in these galaxies has a stellar origin (Irwin \& Sarazin 1998). Power law ellipticals tend to be non-central in groups/clusters and therefore tidally subordinate with some exceptions. More luminous group-centered, group-dominant ellipticals typically have much larger $L_{x} / L_{B}$, enhanced by an additional contribution of circumgalactic or intragroup hot gas (Helsdon et al. 2001; Matsushita 2001). The possible influence of galactic rotation and (oblate) flattening on $L_{x} / L_{B}$ have been explored with limited success (Nulsen et al. 1984; Kley \& Mathews 1995; Brighenti \& Mathews 1996; Pellegrini, Held \& Ciotti 1997; D'Ercole \& Ciotti 1998). While there is some evidence that $L_{x} / L_{B}$ is several times lower in flattened and rotating ellipticals, this cannot explain the much larger scatter observed. In the evolutionary scheme of Ciotti et al. (1991) the scatter results from a transition from early Type Ia driven winds to cooling inflows, but this scenario (discussed below) produces too much iron in the hot gas (Loewenstein \& Mathews 1991). Mathews and Brighenti (1998) showed that ellipticals with

larger $L_{x}$ also have more extended hot gas, $L_{x} / L_{B} \propto\left(R_{e x} / R_{e}\right)^{0.60 \pm 0.20}$, where $R_{e x}$ is the half-brightness radius for the X-ray image which can extend out to $\sim 10 R_{e}$ or beyond. This correlation (see also Fukugita \& Peebles 1999 and Matsushita 2001) may result from the tidal competition for diffuse baryonic gas among elliptical-dominated galaxy groups as they formed with different degrees of relative isolation. Ram pressure stripping must also influence $L_{x} / L_{B}$ for $\mathrm{E}$ galaxies in richer clusters (e.g. Toniazzo \& Schindler 2001).

\section{HYDROSTATIC EQUILIBRIUM AND MASS DETERMINA- TIONS}

Evidence for dark halos in elliptical galaxies from stellar velocities has been slow in coming because of uncertainties in geometrical projection and in the anisotropy of stellar orbits as well as the inherent faintness of starlight beyond $R_{e}$ where dark matter may begin to dominate the potential. Nevertheless, recent optical studies have revealed the presence of outwardly increasing mass to light ratios consistent with a dark matter contribution that is appreciable but not dominant at $\sim R_{e}$ (Saglia et al 1992; Carollo et al 1995; Rix et al 1997; Gerhard et al 1998; Emsellem et al. 1999; Matthias \& Gerhard 1999). Gravitational lensing of background objects provides independent evidence for a dark matter component in elliptical galaxies and their surrounding groups (e.g. Keeton 2001). Fortunately, the X-ray emitting hot gas allows in principle a much better determination of the total mass profile to very large radii. Accurate mass determinations require high quality Xray observations of the gas density and (especially) temperature profiles plus reasonable assurance that the gas is in hydrostatic equilibrium and that gas pressure dominates. The mass distribution can only be determined for rather massive E galaxies, $L_{B} \gtrsim L_{B, c r i t}$, in which the X-ray emission is dominated by gas, not stellar sources. 
Hydrostatic equilibrium requires that systematic and turbulent hot gas velocities are subsonic. Information moving at the sound speed in the hot gas in E galaxies, $(\gamma P / \rho)^{1 / 2} \sim 513 T_{\mathrm{keV}}^{1 / 2} \mathrm{~km} \mathrm{~s}^{-1}$, crosses the optical half-light radius $R_{e} \sim 10 \mathrm{kpc}$ in only $t_{s c} \sim 2 \times 10^{7}$ years. If the hot gas is losing energy by radiative losses, it should flow inward at a rate $\dot{M} \sim L_{x, b o l} /\left(5 k T / 2 \mu m_{p}\right) \approx 1.5$ $M_{\odot} \mathrm{yr}^{-1}$ where $L_{x, b o l} \sim 5 \times 10^{41} \mathrm{erg} \mathrm{s}^{-1}$ is a typical X-ray bolometric luminosity for massive E galaxies. Using $n_{e}\left(R_{e}\right) \sim 0.01 \mathrm{~cm}^{-3}$ for a typical hot gas density at $R_{e}$, the systematic inflow velocity, $u \sim \dot{M} / n_{e}\left(R_{e}\right) m_{p} 4 \pi R_{e}^{2} \sim 5 \mathrm{~km} \mathrm{~s}^{-1}$, is highly subsonic, consistent with hydrostatic equilibrium. Less is known about the magnitude of turbulent motions in the hot gas, but radial velocities of diffuse optical emission lines from gas at $T \sim 10^{4} \mathrm{~K}$ in the central regions of E galaxies (e.g. Caon et al. 2000), $v_{\text {turb }} \lesssim 150 \mathrm{~km} \mathrm{~s}^{-1}$, suggest subsonic motion, assuming this cold gas comoves with the local hot gas.

The condition for hydrostatic equilibrium $d P_{t o t} / d r=-G M \rho / r^{2}$ allows a direct determination of the total mass of stars and dark matter within each radius:

$$
M(r)=-r T(r) \frac{k}{G \mu m_{p}}\left(\frac{d \log \rho}{d \log r}+\frac{d \log T}{d \log r}+\frac{P_{n t}}{P} \frac{d \log P_{n t}}{d \log r}\right)
$$

where $m_{p}$ is the proton mass and $\mu=0.61$ is the molecular weight for full ionization. In addition to the gas pressure $P$, an additional non-thermal turbulent, magnetic or cosmic ray pressure $P_{n t}$ may be present. In ellipticals containing strong radio sources Faraday depolarization at radio frequencies provides direct evidence for $P_{n t}$ (e.g. Garrington et al. 1988; Garrington \& Conway 1991), but this pressure is usually ignored in most $\mathrm{E}$ galaxy mass determinations. Nonradiating relativistic protons may also be present (e.g. Fabian et al. 2002a), so it is unclear if $P_{n t}$ can always be ignored.

The total integrated mass $M(r)$ can be estimated by using the average temperature within some radius. Loewenstein \& White (1999) studied the ratio of the dimensional coefficients in the equation above, $\beta=\langle\sigma\rangle^{2} /\left(k\langle T\rangle / \mu m_{p}\right)$ where $\langle\sigma\rangle^{2} \propto G M(r) / r$ is the central stellar velocity dispersion (assumed to be isotropic) and $\langle T\rangle$ is the mean hot gas temperature within $6 R_{e}$ determined from fits to the thermal X-ray spectrum. Loewenstein \& White considered an optically complete sample of over 40 E galaxies (Davis \& White 1996). Using accurate stellar mass profiles normalized to the fundamental plane, they determined that $\beta \approx 0.75-1.2$ should be expected in the absence of dark matter. The observed values, $\beta \approx 0.6 \pm 0.1$, clearly require a dark matter component. Both the gas and the dark matter are hotter than the central stars. Loewenstein \& White conclude that dark matter increases from stellar values at the origin $\left\langle\Upsilon_{V}\right\rangle \equiv\left\langle M / L_{V}\right\rangle \approx 10 h_{70} M_{\odot} / L_{V, \odot}$ to $\left\langle\Upsilon_{V}\right\rangle \approx 22 h_{70} M_{\odot} / L_{V, \odot}$ within $6 R_{e}$. Extended dark halos are a common property of all bright ellipticals.

For a few bright E galaxies both $T(r)$ and $n_{e}(r)$ can be determined and Equation (1) can be solved directly for the total mass profile $M(r)$. Figure 2a shows the electron density profile in NGC 4472, a well-observed massive E1 galaxy and the brightest galaxy in the Virgo cluster, at an assumed distance of $\mathrm{d}=17 \mathrm{Mpc}$ $\left(n_{e} \propto d^{-1 / 2}\right)$. The hot gas density profiles may have small flattened cores but vary as $n_{e} \propto r^{-p}$ at larger radii with $p \approx 1-1.5$, so the gas mass increases outward. Using Einstein HRI data Trinchieri, Fabbiano \& Canizares (1986) showed that the optical and X-ray surface brightness profiles are almost identical for three bright Virgo ellipticals, NGC 4649, NGC 4636 and NGC 4472, so that $\rho_{*} \propto n_{e}^{2}$. This remarkable result is illustrated again in Figure $2 \mathrm{a}$ where $n_{e} \propto \rho_{*}^{1 / 2}$ is seen 
to hold over a wide range in galactic radius.

Figure $2 \mathrm{~b}$ shows the hot gas temperature profiles for several massive E galaxies. The temperature in these group-dominant $\mathrm{E}$ galaxies rises from a minimum value near the galactic center to a maximum at several $R_{e}$ and, if the gas is sufficiently extended, is either uniform or slowly decreasing beyond (Brighenti $\&$ Mathews 1997 and references therein), sometimes extending to $\gtrsim 10 R_{e}$. (In cluster-centered E galaxies the temperature continues to rise to the cluster gas temperature.) The radiative cooling time at constant pressure in the hot gas in NGC 4472 is quite short, $t_{c o o l} \approx 10^{8} r_{k p c}^{1.2}$ yrs, but greater than the dynamical time $t_{d y n} \approx 3 \times 10^{6} r_{k p c}^{0.85}$ yrs. The entropy factor $T n^{-2 / 3}$ for NGC 4472 is relatively flat within $r \sim 0.55 \mathrm{kpc}$, suggesting local heating (David et al. 2001), then increases monotonically with radius, $T n^{-2 / 3} \approx 6.5 \times 10^{7} r_{k p c}^{0.8712} \mathrm{~K} \mathrm{~cm}^{2}$, as required for convective stability. Recent Chandra observations often show surface brightness fluctuations and cavities, sometimes extending to $\sim R_{e}$, that suggest deviations from hydrostatic equilibrium.

The total mass $M_{\text {tot }}(r)$ profile for NGC 4472 determined from Equation (1) (with $P_{n t}=0$ and data from Figs. 2a and 2b) is plotted in Figure 3a. Also shown is the stellar mass distribution $M_{*}(r)$ based on a de Vaucouleurs profile $\rho_{*, d e V}(r)$ (total mass: $M_{* t}=7.26 \times 10^{11} M_{\odot}$; effective radius: $R_{e}=1.733^{\prime}=8.57 \mathrm{kpc}$ ) with a core $\rho_{*, \text { core }}(r)=\rho_{*, d e V}\left(r_{b}\right)\left(r / r_{b}\right)^{-0.90}$ within the break radius $r_{b}=2.41^{\prime \prime}=200$ pc (Gebhardt et al. 1996; Faber et al. 1997). It is remarkable that the total mass $M_{t o t}(r)$ in Figure 3a determined with Equation (1) agrees quite well with the de Vaucouleurs mass profile in the range $0.1 \lesssim r / R_{e} \lesssim 1$. The best fitting stellar profile corresponds to a mass to light ratio of $\Upsilon_{B} \equiv M / L_{B} \approx 7$, slightly less than $\Upsilon_{B}=9.2$ determined for NGC 4472 from axisymmetric stellar models near the galactic core (van der Marel 1991). This consistency of X-ray and stellar mass profiles suggests that the stellar mass to light ratio in NGC 4472 does not change greatly with galactic radius in $0.1 \lesssim r / R_{e} \lesssim 1$ (Brighenti \& Mathews 1997a; also for NGC 720: Buote et al. 2002a). As X-ray observations improve we expect that they will provide much information on the stellar mass to light ratio for $r \lesssim R_{e}$. At small radii $r \lesssim 0.03 R_{e}$ in NGC $4472, M_{t o t}$ is less than $M_{*}$. This may indicate some additional non-thermal pressure $P_{n t}$ in this region or a deviation from hydrostatic equilibrium. Like most bright E galaxies, NGC 4472 contains a faint double lobe radio source that extends to $\sim 0.5 R_{e}$ (Ekers \& Kotanyi 1978).

The dark halo mass clearly dominates in Figure 3a for $r \gtrsim R_{e}$ where $M_{t o t}(r)$ rises sharply above the de Vaucouleurs profile (e.g. Brighenti \& Mathews 1997a; Kronawitter et al. 2000). The shape of the dark halo is consistent with an NFW halo (Navarro, Frenk \& White 1996), but the virial mass of the dark halo surrounding NGC 4472 and its mass profile are poorly determined in part due to uncertainties in the hot gas temperature beyond several $R_{e}$. In addition, the X-ray image of NGC 4472 is asymmetric for $r \gtrsim 2.5 R_{e}$, as seen in Figure 4, apparently because of its motion through the more extended Virgo cluster gas or possibly due to its interaction with the nearby dwarf irregular galaxy UGC 7636 (Irwin \& Sarazin 1996; 1997). (See Fabbiano et al. 1992 for an atlas of similar figures.) In spite of these problems, the azimuthally averaged gas density profile around NGC 4472 is similar to the mean profile of about 10 other bright $\mathrm{E}$ galaxies out to at least $18 R_{e}$.

In non-spherical E galaxies, the existence of massive dark halos can be inferred directly from the X-ray image independent of the hot gas temperature profile - 

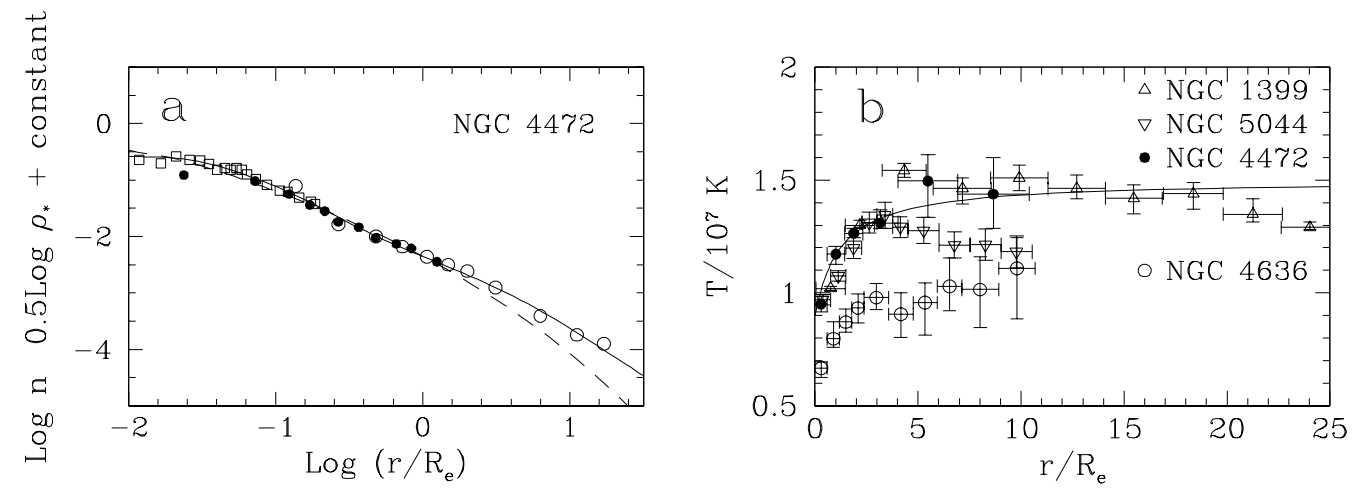

Figure 2: (2a Left panel:) The observed and azimuthally averaged electron density profile $n(r)$ in NGC 4472 is shown as a function of radius normalized to the effective radius $R_{e}=8.57 \mathrm{kpc}$ at distance $d=17 \mathrm{Mpc}$. The observations are from Einstein (Trinchieri, Fabbiano, \& Canizares 1986) (filled circles) and ROSAT (Irwin \& Sarazin 1996) (open circles); for the inner region we have Abel-inverted Chandra surface brightness data from Loewenstein et al. (2001) (open squares) and normalized them to previous observations. The solid line is an analytic fit to the observations. The dashed line is the square root of the stellar density $\rho_{*}^{1 / 2}(r)$ normalized to $n$ at $r=R_{e}$. (2b Right panel:) Typical temperature profiles for several bright E galaxies, taken from Brighenti \& Mathews (1997a), based on the following sources: NGC 1399: ROSAT PSPC from Jones et al. (1997); NGC 5044: ROSAT PSPC from David et al. (1994); NGC 4636: ROSAT PSPC from Trinchieri et al. (1994); NGC 4472: ROSAT HRI AND PSPC from Irwin \& Sarazin (1996). The solid line is an approximate analytic fit to $T(r)$ for NGC 4472. 

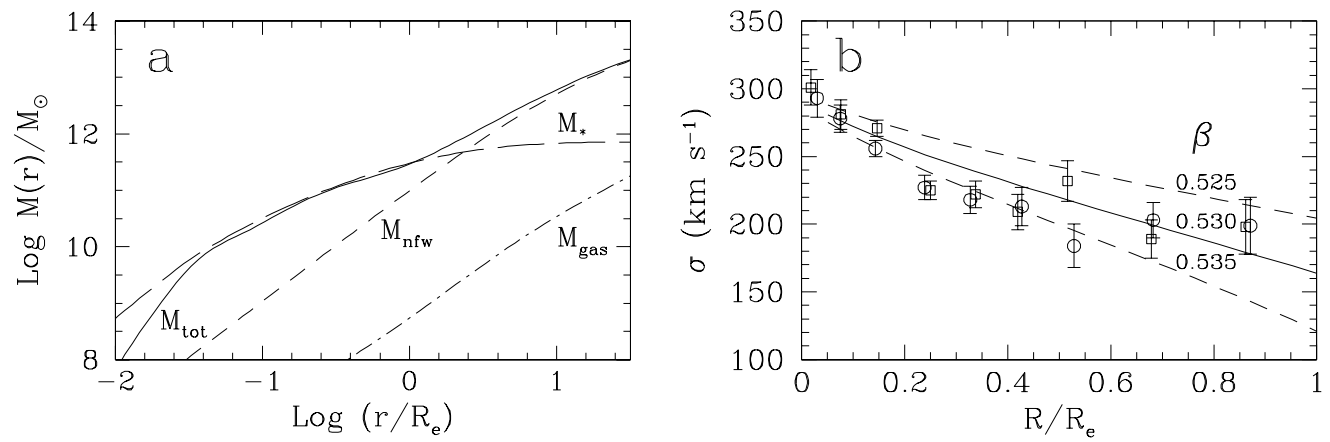

Figure 3: (3a Left panel:) The total mass $M_{\text {tot }}(r)$ for NGC 4472 (solid line) is found from Equation (1) with $P_{n t}=0$ using the solid line approximations to the X-ray observations in Figure 2. The total mass of hot gas $M_{g a s}(r)$ (dotdashed line) is relatively small. The stellar mass profile $M_{*}(r)$ (long dashed line) is based on a de Vaucouleurs plus core profile with mass to light ratio $\Upsilon_{B}=7$. The NFW dark halo profile $M_{n f w}(r)$ (short dashed line) corresponds to a total mass of $4 \times 10^{13} M_{\odot}$. (3b Right panel:) Line of sight stellar velocity dispersion profiles $\sigma(\beta ; R)$ as a function of projected radius $R$. The curves are computed from solutions $\sigma_{r}(r)$ of Equation (2) assuming constant $\beta$ which labels each curve. The observations are from Fried \& Illingworth (1994). 


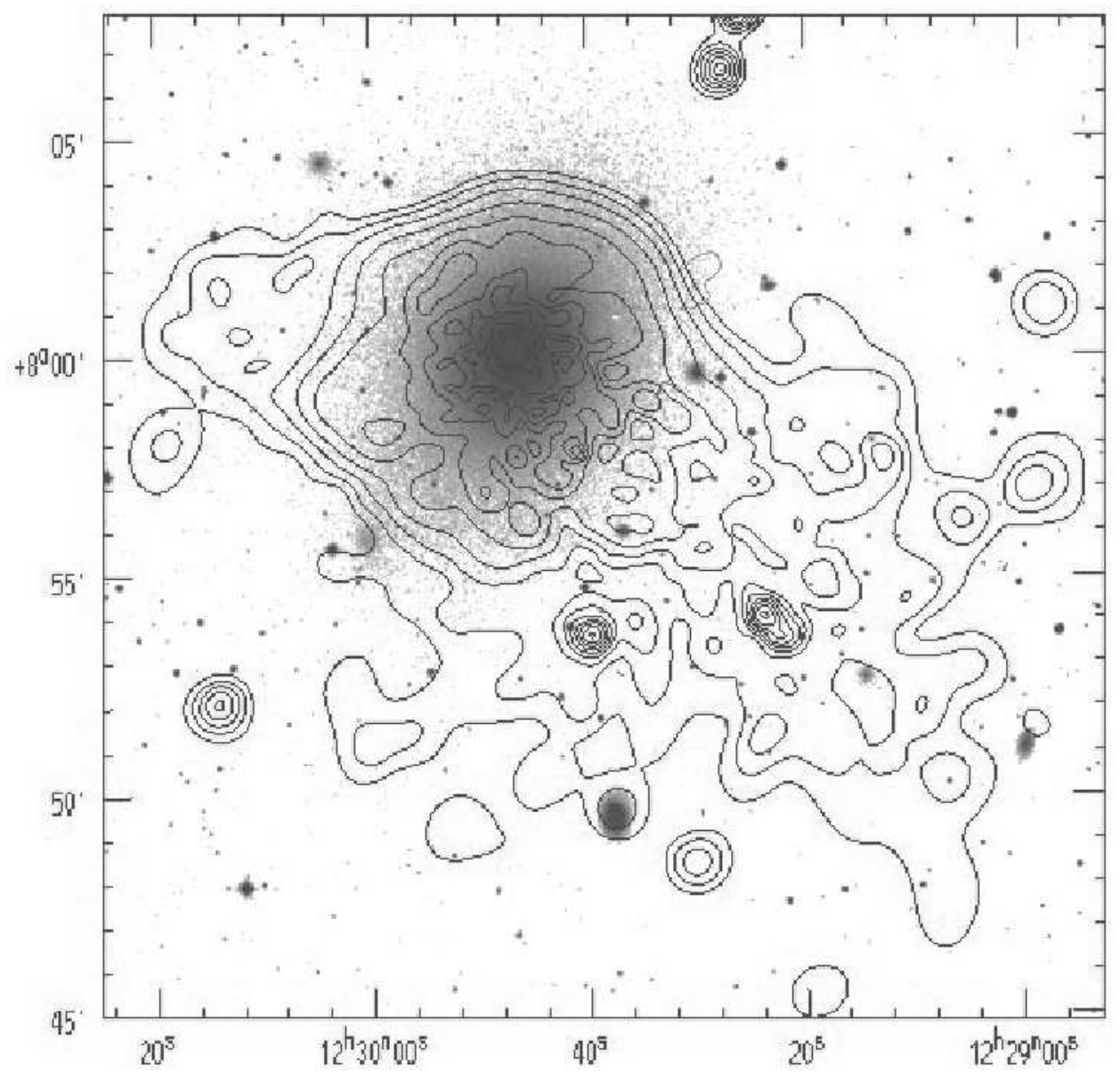

Figure 4: Contours show a combined ROSAT HRI and PSPC X-ray image of NGC 4472 superimposed on an optical image from the Digital Sky Survey (Irwin \& Sarazin 1996). 
providing the gas is in hydrostatic equilibrium and rotation has little influence on the potential. For example, Buote \& Canizares (1998) find that the X-ray isophotes of the E4 galaxy NGC 3923 have ellipticity $\epsilon_{x}=0.15 \pm 0.05$, which is significantly less than that in the R-band, $\epsilon_{R}=0.30$. Although the gravitational potential is always more spherical than the mass distribution, Buote \& Canizares show that this $\epsilon_{x}$ can be understood only if the confining mass is greater and more extended than any mass distribution proportional to the optical light (also: Buote et al. 2002a). Furthermore, the dark mass must have an ellipticity $\epsilon_{d m}=$ $0.35-0.66$ greater than the optical image. Not only is this an elegant method to detect dark matter (see also Buote \& Canizares 1996; 1997), it serves as a warning, generally ignored, that the dark matter may not be distributed in a quasi-spherical fashion.

While the hot gas in NGC 4472, NGC 4649 and NGC 720 appears to be in hydrostatic equilibrium in the stellar potential in $0.1 \lesssim r / R_{e} \lesssim 1$, this circumstance may not be universal. For example, in NGC 4636 Brighenti \& Mathews (1997a) found that the total mass $M(r)$ profile found from Equation (1) (with $P_{n t}=0$ ) intersects the stellar mass $M_{*}(r)$ (using $\Upsilon_{B}=10.7$ ) with no slope change whatsoever. Brighenti \& Mathews argued that this insensitivity may be due to a magnetic field, $B \sim 100 \mu \mathrm{G},\left(P_{n t} \approx B^{2} / 8 \pi\right)$ at $r \sim 0.1 R_{e}$, so that the missing $\left(P_{n t} / P\right)\left(d \log P_{n t} / d \log P\right)$ term in Equation (1) would account for the discrepancy. Cosmic rays or energetic turbulence would serve equally well. In fact the Chandra X-ray image of NGC 4636 shows that the hot gas is strongly agitated for $r \lesssim R_{e}$ (Jones et al. 2002), consistent with a breakdown in hydrostatic equilibrium. Alternatively, in reconciling the total mass $M(r)$ of NGC 4636 from X-ray data, Loewenstein \& Mushotzky (2002) reduced the contribution of the stars by lowering the stellar mass to light ratio to $\Upsilon_{B}<5.4$ (at $d=17 \mathrm{Mpc}$ ) which is very much less than values determined for NGC 4636 from stellar velocities: $\Upsilon_{B}=10.7$ (van der Marel 1991) or $\Upsilon_{B}=11.3$ (Kronawitter et al. 2000) (both at $d=17 \mathrm{Mpc}$ ).

The shape of the stellar velocity ellipsoid $\beta(r)$ can also be estimated directly from X-ray observations, especially for very massive E galaxies that are approximately spherical. Here $\beta(r)=1-\sigma_{t}^{2} / \sigma_{r}^{2}$ depends on $\sigma_{r}$ and $\sigma_{t}=\sigma_{\theta}=\sigma_{\phi}$, the velocity dispersions in the radial and transverse directions. Combining the Jeans Equation for the radial stellar velocity dispersion $\sigma_{r}$ with the equation for hydrostatic equilibrium in the gas we find

$$
\frac{d \sigma_{r}^{2}}{d r}+\frac{\sigma_{r}^{2}}{r}\left[\frac{d \log \rho_{*}}{d \log r}+2 \beta\right]=\frac{d c^{2}}{d r}+\left[\frac{c^{2}}{r} \frac{d \log \rho}{d \log r}\right]
$$

where $c^{2}=k T / \mu m_{p}$ is the isothermal sound speed. From Figure 2a for NGC 4472 we see that $0.5\left(d \log \rho_{*} / d \log r\right)=d \log \rho / d \log r=-1.18$ fits over $-1.3 \lesssim$ $\log \left(r / R_{e}\right) \lesssim 0$. The gas temperature variation is approximately linear over this region, $c^{2}(r) \approx 4135\left(r_{k p c}+30\right)\left(\mathrm{km} \mathrm{s}^{-1}\right)^{2}$ (Figure $\left.2 \mathrm{~b}\right)$. If $\beta$ is assumed to be constant, Equation (2) can be solved analytically for $\sigma_{r}^{2}(r)$ and the line of sight stellar velocity dispersion as a function of projected radius $\sigma(\beta ; R)$ can be found by integration (e.g. Binney \& Mammon 1982). The resulting $\sigma(\beta ; R)$, when compared with stellar velocity dispersion observations in Figure $3 \mathrm{~b}$, suggests $\beta=$ $0.530 \pm 0.005$, somewhat higher than that of Kronawitter et al. (2002) who use different velocity data. Conversely, if $\beta(r)$ is known securely from stellar data, Equation (2) can be used to determine the gas temperature profile $c^{2}(r)$. 


\section{GAS DYNAMICAL EQUATIONS AND CLASSIC COOL- ING FLOWS}

To understand cooling flows at the next level beyond static models, the assumption of steady state flow is often made (Bailey 1980; White \& Chevalier 1983, 1984; Nulsen, Stewart \& Fabian 1984; Thomas 1986; Sarazin \& White 1987; Vedder, Trester \& Canizares 1988; Sarazin \& Ashe 1989; Tabor \& Binney 1993; Bertin \& Toniazzo 1995). Although the steady state approximation is useful in gaining insight into various dynamical aspects of subsonic cooling flows, particularly at small galactic radii, inconsistencies can arise if this approximation is applied globally. In particular for subsonic flows there are ambiguities in selecting the boundary conditions near the stagnation radius where the inward integrations begin, as recognized and discussed by Vedder, Trester \& Canizares (1988). Steady flows are also incapable of properly allowing for some essential time dependencies such as the strongly decreasing rate of stellar mass loss, variations in the frequencies of Type Ia supernovae, heating by central AGN, and the accretion of intergalactic gas in an evolving cosmology. For these reasons we emphasize non-steady flows in the following discussion.

For galactic scale cooling flows, the usual time-dependent gas dynamical equations must include appropriate source and sink terms:

$$
\begin{gathered}
\frac{\partial \rho}{\partial t}+\nabla \cdot \rho \mathbf{u}=\alpha \rho_{*} \\
\rho \frac{d \mathbf{u}}{d t}=\rho\left[\frac{\partial \mathbf{u}}{\partial t}+(\mathbf{u} \cdot \nabla) \mathbf{u}\right]=-\nabla P-\rho \nabla \Phi-\alpha \rho_{*}\left(\mathbf{u}-\mathbf{u}_{*}\right),
\end{gathered}
$$

and

$$
\rho \frac{d \varepsilon}{d t}-\frac{P}{\rho} \frac{d \rho}{d t}=-\frac{\rho^{2} \Lambda}{m_{p}^{2}}+\alpha \rho_{*}\left[\varepsilon_{o}-\frac{P}{\rho}-\varepsilon+\frac{1}{2}\left|\mathbf{u}-\mathbf{u}_{*}\right|^{2}\right]-\nabla \cdot \mathbf{F}_{\text {cond }}+H(r, t) \rho
$$

where $\varepsilon=3 k T / 2 \mu m_{p}$ is the specific thermal energy. The gravitational potential $\Phi$ in the momentum Equation (4) includes contributions from both stellar and dark mass; the mass of hot gas is usually negligible. The coefficient $\alpha=\alpha_{*}+\alpha_{s n}$ is the specific rate of mass loss from the stars and Type Ia supernovae, i.e. $\alpha M_{* t} \approx$ $0.15 M_{* t} /\left(10^{11} M_{\odot}\right) M_{\odot} \mathrm{yr}^{-1}$ is the total current rate of mass loss from evolving stars in NGC 4472. Because $\alpha_{s n}$ is much less than $\alpha_{*}, \alpha \approx \alpha_{*}$ is an excellent approximation. The specific mass loss rate from a single burst stellar population with Salpeter IMF and age $t$ varies as $\alpha_{*}(t)=4.7 \times 10^{-20}\left(t / t_{n}\right)^{-1.3} \mathrm{~s}^{-1}$ where $t_{n}=13$ Gyrs (Mathews 1989); for other powerlaw IMFs $\alpha_{*}\left(t_{n}\right)$ varies inversely with the stellar mass to light ratio.

A fundamental assumption is that gas ejected from evolving giant stars as winds or planetary nebulae eventually becomes part of the hot phase. The interaction of gas ejected from orbiting stars with the hot galactic gas is extremely complicated, involving complex hydrodynamic instabilities that enormously increase the surface area between the ejected gas and its hot environment until, as usually assumed, the two gases thermally fuse. As we discuss below, the positive temperature gradients observed in inner cooling flows of cluster-centered elliptical galaxies and the hot gas oxygen abundance gradients provide indirect evidence that such mixing does occur. The term $\alpha \rho_{*}\left(\mathbf{u}-\mathbf{u}_{*}\right)$ in Equation (4) represents a drag on the flow, assuming gas expelled from stars has on average 
the mean stellar velocity $\mathbf{u}_{*}$, which is non-zero only in rotating galaxies. This drag term is generally negligible if the flow is subsonic. If $u_{*}=0$, this term has the effect of making subsonic flow even more subsonic and supersonic flow even more supersonic.

The thermal energy Equation (5) contains a term $-\left(\rho / m_{p}\right)^{2} \Lambda(T, z)$ for the loss of energy by X-ray emission; the radiative cooling coefficient $\Lambda(T, z)$ erg $\mathrm{cm}^{3} \mathrm{~s}^{-1}$ varies with both gas temperature and metal abundance (e.g. Sutherland \& Dopita 1993). The dissipative heating $\alpha_{*} \rho_{*}\left|\mathbf{u}-\mathbf{u}_{*}\right|^{2} / 2$ involved in accelerating stellar ejecta to the local flow velocity is usually very small. The hot gas temperature is also influenced by stellar mass loss and Type Ia supernovae. The source terms $\alpha_{*} \rho_{*}\left(\varepsilon_{o}-P / \rho-\varepsilon\right)$ represent the heating of the hot interstellar gas of specific energy $\varepsilon$ by the mean energy of stellar ejecta $\varepsilon_{o}$ less the work done $P / \rho$ in displacing the hot gas. The mean gas injection energy is $\varepsilon_{o}=3 k T_{o} / 2 \mu m_{p}$ where $T_{o}=\left(\alpha_{*} T_{*}+\alpha_{s n} T_{s n}\right) / \alpha$. The stellar temperature $T_{*}$ can be found by solving the Jeans equation, but this term is small and it is often sufficient to use an isothermal approximation, $T_{*}=\left(\mu m_{p} / k\right) \sigma^{2}$, where $\sigma$ is the average stellar velocity dispersion. Supernova heating is assumed to be distributed smoothly in the gas, ignoring the detailed evolution of individual blast waves (Mathews 1990). The heating by Type Ia supernovae, each of energy $E_{s n} \approx 10^{51} \mathrm{ergs}$, is described by multiplying the characteristic temperature of the mass $M_{s n}$ ejected, $T_{s n}=2 \mu m_{p} E_{s n} / 3 k M_{s n}$, by the specific mass loss rate from supernovae, $\alpha_{s n}=3.17 \times 10^{-20} \mathrm{SNu}(t)\left(M_{s n} / M_{\odot}\right) \Upsilon_{B}^{-1} \quad \mathrm{~s}^{-1}$. Here the supernova rate $\mathrm{SNu}$ is expressed in the usual $\mathrm{SNu}$-units, the number of supernovae in 100 yrs expected from stars of total luminosity $10^{10} L_{B \odot}$.

Supernovae in ellipticals today are infrequent and all of Type Ia. Cappellaro et al. (1999) find $\mathrm{SNu}\left(t_{n}\right)=(0.16 \pm 0.05) h_{70}^{2}$ for $\mathrm{E}+\mathrm{S} 0$ galaxies. The past evolution of this rate $\mathrm{SNu}(t)$ is unknown, although some provisional data is beginning to emerge (Gal-Yam, Maoz, \& Sharon 2002). Clearly, it would be very useful to have more information about $\mathrm{SNu}(t)$ for E galaxies at high redshift because this can have a decisive influence on the evolution of the hot gas and its iron abundance. Type Ia supernovae may involve mass exchange between binary stars of intermediate mass, but the details are very uncertain (e.g. Hillebrandt et al. 2000). However, like all cosmic phenomena, it is generally assumed that $\mathrm{SNu}(t)$ is a decreasing function of time, $\mathrm{SNu}(t)=\mathrm{SNu}\left(t_{n}\right)\left(t / t_{n}\right)^{-s}$. This is consistent with measurements at intermediate redshifts if $s \sim 1$ (Pain et al. 2002), although these observations refer to all galaxy types.

Ciotti et al. (1991) recognized that the relative rates of stellar mass ejection $\left(\alpha_{*} \sim t^{-1.3}\right)$ and Type Ia supernova $\left(\mathrm{SNu} \sim t^{-s}\right)$ determine the dynamical history of the hot interstellar gas in ellipticals. For example, if $s>1.3$ in isolated ellipticals then the supernova energy per unit mass of gas expelled from stars $\left(\propto \alpha_{s n} / \alpha_{*}\right)$ was large in the distant past, promoting early galactic winds, but if $s<1.3$, outflows or winds tend to develop at late times. However, in the presence of circumgalactic gas, the early time galactic winds driven by Type Ia supernovae can be suppressed. Each Type Ia supernova injects $\sim 0.7 M_{\odot}$ of iron into the hot gas, producing a negative iron abundance gradient in the hot gas that depends on $\rho_{*} / \rho$ and the radial flow velocity of the gas. The observed SNIa enrichment provides an important constraint on $d \mathrm{SNu}(t) / d t$ (Loewenstein \& Mathews 1991). Evolutionary flow solutions with $s>1.3$ produce iron abundances far in excess of those observed today, unless the iron is preferentially removed by selective cooling. We therefore have tentatively adopted $s=1$ with $\mathrm{SNu}\left(t_{n}\right)=0.06 \mathrm{SNu}$ 
(similar to the estimate of Kobayashi et al 2000) although this is by no means the only possibility. This adopted current Type Ia supernova rate is less than the rate observed in E plus S0 galaxies, but the rate may be lower for ellipticals than for S0 galaxies.

Thermal conductivity in a hot plasma, $\kappa \approx 5.36 \times 10^{-7} T^{5 / 2} \mathrm{erg} \mathrm{s}^{-1} \mathrm{~cm}^{-1} \mathrm{~K}^{-1}$ is important at high temperatures, but may be reduced by tangled magnetic fields. The conductive energy flux in Equation (5), $F_{\text {cond }}=f \kappa d T / d r$, usually includes an additional factor $f \leq 1$ to account for magnetic suppression. In the past $f \ll 1$ has often been assumed, but Narayan \& Medvedev (2001) have recently shown that $f \sim 0.2$ is appropriate for thermal conduction in a hot plasma with chaotic magnetic field fluctuations. The final term in Equation $(5), H(r, t) \rho$, is an ad hoc AGN heating term that is discussed later.

Using the relation $\rho_{*} \approx 8.54 \times 10^{-20} n_{e}^{2}$ from Figure $2 \mathrm{a}$, we find at the current time $t_{n}$ that $\left(\rho / m_{p}\right)^{2} \Lambda$ is about an order of magnitude greater than $\alpha_{*} \rho_{*}\left(\varepsilon_{o}-\right.$ $P / \rho-\varepsilon)$. For galactic flows with $T \sim 10^{7} \mathrm{~K}$ thermal conduction is important for $f \gtrsim 0.5$. Therefore, if dissipation and AGN heating are small in NGC 4472, $\left(\rho / m_{p}\right)^{2} \Lambda$ dominates all other non-adiabatic terms in Equation (5), generating a classic cooling inflow driven by radiative losses. As radiative energy is lost in a Lagrangian frame moving with the gas, the entropy decreases, but the gas temperature $\sim T_{\text {vir }}$ remains relatively constant as the gas is heated in the gravitational potential by $P d v$ compression. The compression drives gas slowly toward the galactic center where, in this simple example, $P d v$ heating is no longer available and catastrophic cooling ensues. Because of this self-regulating mechanism, the temperature profile $T(r)$ in cooling flows is very insensitive to modest changes in the source terms in the thermal energy Equation (5), including $\left(\rho / m_{p}\right)^{2} \Lambda$.

To gain further insight, it is instructive to insert the observed gas density and temperature profiles for NGC 4472 into Equations (3) and (5) and estimate the steady state radial gas velocity, assuming $\alpha_{*}$ is fixed at its current value. For a steady inflow Equation (3) can be integrated from $r$ to $\infty$ and solved for the flow velocity, $u_{\alpha}(r)=\left\{M(\infty)-\alpha_{*}\left[M_{* t}-M_{*}(r)\right]\right\} / 4 \pi r^{2} \rho$. This is the negative velocity required to continuously remove mass supplied by mass-losing galactic stars without changing $\rho(r)$. Assuming $\dot{M}(\infty) \approx 0$ and using NGC 4472 parameters, we find $u_{\alpha}(r) \approx-51 r_{k p c}^{-1.05} \mathrm{~km} \mathrm{~s}^{-1}$ for $0.25 \lesssim r_{k p c} \lesssim 10$; at larger $r$ in NGC 4472 the inflow of circumgalactic gas $\dot{M}(\infty)<0$ must be considered but for $r \lesssim R_{e}=8.57 \mathrm{kpc}$ we can assume the mass lost from the stars determines $\rho(r)$. Another steady state velocity can be found by inserting the observed $\rho(r)$ and $T(r)$ (Fig. 2) into the first three terms of Equation (5),

$$
u_{\Lambda}(r)=-\left(\frac{3}{2} \frac{d \log T}{d \log r}-\frac{d \log \rho}{d \log r}\right)^{-1} \frac{\mu}{k} \frac{\rho}{m_{p}} \frac{r \Lambda(T)}{T} .
$$

This "slump" velocity in NGC 4472, $u_{\Lambda}(r) \approx-27 r_{k p c}^{-0.36} \mathrm{~km} \mathrm{~s}^{-1}$ for $0.3 \lesssim r_{k p c} \lesssim$ 40 , is the inflow that occurs because gas is cooling near the center and occupying less volume. Both $u_{\alpha}$ and $u_{\Lambda}$ are very small compared to the adiabatic sound speed in the hot gas, $476\left(T / 10^{7} \mathrm{~K}\right)^{1 / 2} \mathrm{~km} \mathrm{~s}^{-1}$, so either type of flow satisfies the requirement for hydrostatic equilibrium. However, for $r_{k p c} \lesssim 2.5,\left|u_{\alpha}\right|>\left|u_{\Lambda}\right|$, i.e., the inflow required to conserve the observed gas density profile exceeds the rate that the gas can cool, violating the assumption of steady flow. Consequently, if the observed $\rho(r)$ and $T(r)$ are taken as initial conditions in a time-dependent gasdynamical calculation for NGC 4472, subsonic inflowing solutions evolve to- 
ward higher gas densities near the origin, increasing the radiative losses there until $u_{\alpha}(r) \approx u_{\Lambda}(r)$. This explains why the central gas density exceeds observed values in every otherwise successful steady state or time-dependent inflow model without central AGN heating or thermal conduction.

\section{SIMPLE COOLING FLOW MODELS}

To illustrate the influence and relevance of various terms in Equations (3) - (5), we briefly describe several simple time-dependent solutions (also see Loewenstein \& Mathews 1987). For a reference flow we consider an E0 galaxy with no conductive or AGN heating and no source of gas except that lost from the stars, i.e. an "isolated" elliptical galaxy. The calculations begin at cosmic time $t_{i n}=1 \mathrm{Gyr}$ when we imagine that the (recently assembled) galaxy has just been cleared of gas by Type II supernovae. We assume NGC 4472 parameters with an NFW group halo of mass $M_{h}=4 \times 10^{13} M_{\odot}$ and supernova rate $\mathrm{SNu}(t)=\mathrm{SNu}\left(t_{n}\right)\left(t / t_{n}\right)^{-s}$ with $\mathrm{SNu}\left(t_{n}\right)=0.06$ and $s=1$. In this reference model gas cools only at the galactic center and for simplicity we ignore the (not insignificant) gravitational influence of the cooled gas on hot gas near the origin; with this assumption the solutions are less sensitive to $t_{i n}$. After the flow evolves to time $t_{n}=13$ Gyrs we compare the density and temperature profiles with those of NGC 4472 and consider the mass that has cooled and the iron abundance in the hot gas. The stellar iron abundance is $z_{*, F e}=0.75\left[1+\left(r / R_{e}\right)^{2}\right]^{-0.2}$ (in solar meteoritic units) and $0.7 M_{\odot}$ of iron is contributed by each Type Ia supernova. Then we describe the effect on the reference solution when one of the many terms and parameters in Equations (3) - (5) is altered. None of these models agrees completely with the observations although some agree much better than others.

Reference Flow: The reference model at time $t_{n}$, shown with solid lines in column 1 of Figure 5, clearly has a steeper density slope than the observed profile and the temperature beyond about $5 \mathrm{kpc}$ is too low. Both of these discrepancies occur in large part because our reference model galaxy is "isolated". The real NGC 4472 galaxy is (or was) surrounded by an extended circumgalactic cloud of gas at the somewhat higher virial temperature of the dark matter potential of the galaxy group from which NGC 4472 formed. As we discussed earlier, the excess gas density inside $r \sim 10 \mathrm{kpc}$ is a characteristic feature of all flow models except those with additional non-thermal pressure or heating. The temperature gradient $d T / d r$ is negative in disagreement with observations of NGC 4472 and other similar galaxies (Figure 2b). Negative $d T / d r$ occurs because of the steepness of the stellar potential in $r \lesssim R_{e}$. When the reference calculation is repeated ignoring the stellar gravity but retaining only the softer NFW potential, $T(r)$ passes through a maximum around $r \sim 40 \mathrm{kpc}$ and $d T / d r>0$ within this radius, similar to cooling flow thermal profiles in rich clusters. For the reference flow we find $L_{x, b o l}\left(t_{n}\right)=6.7 \times 10^{41} \mathrm{ergs} \mathrm{s}^{-1}$ from the hot diffuse gas. The iron abundance in the hot gas at $r \sim 10 \mathrm{kpc}, z_{\mathrm{Fe}} / z_{\mathrm{Fe}, \odot} \sim 2.7$ (meteoritic), is slightly higher than observed in the hot gas of NGC 4472, $z_{F e} / z_{F e, \odot} \sim 2$ (e.g. Buote et al. 2000a, 2000 c), even though our reference $\mathrm{SNu}\left(t_{n}\right)=0.06$ is rather low.

Circumgalactic Gas: The dashed lines in column 1 of Figure 5 show the effect of including circumgalactic, group-related gas in the NGC 4472 evolution (Thomas 1986; Bertin \& Toniazzo 1995; Brighenti \& Mathews 1998). Agreement with the observations is improved in several ways: (1) By design the gas density 

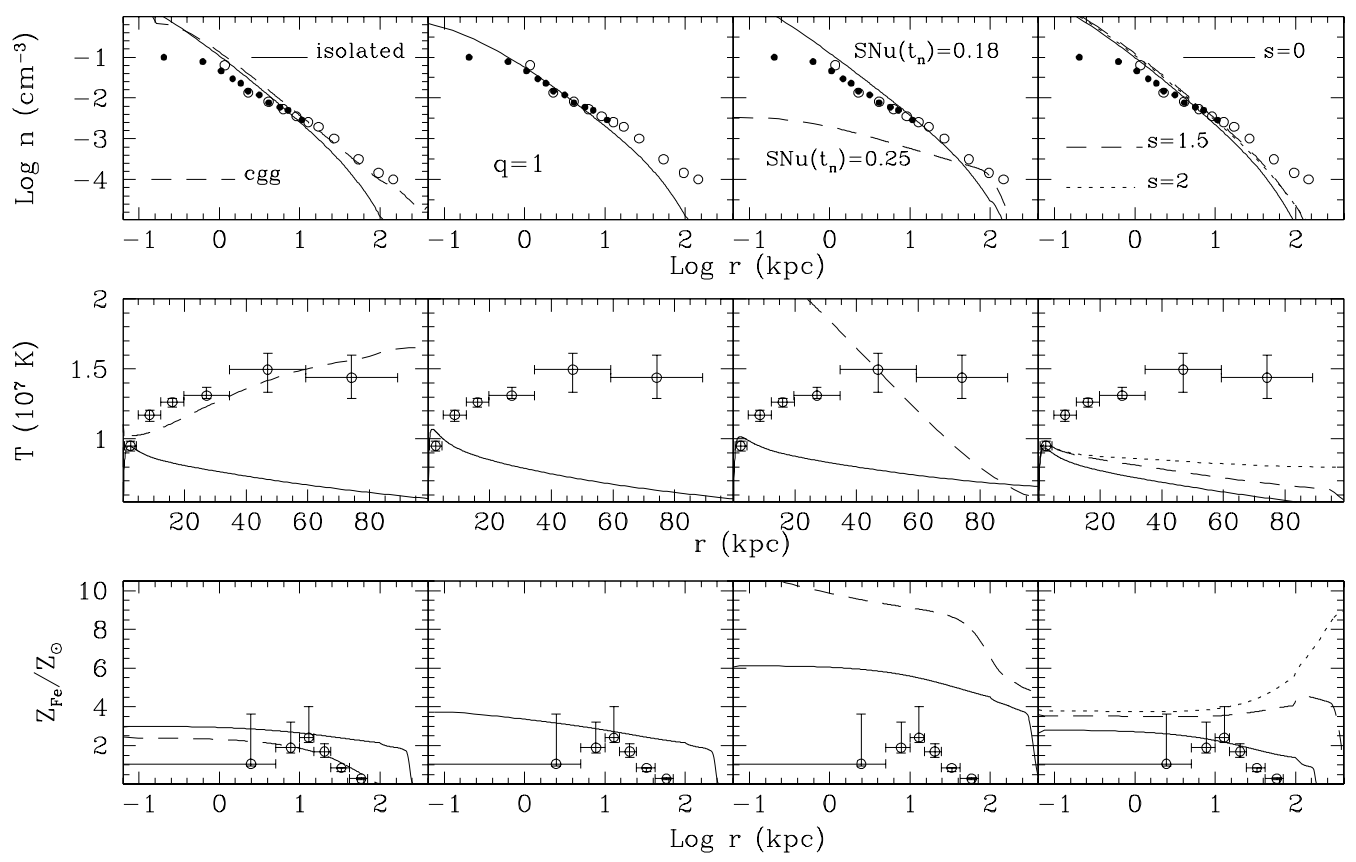

Figure 5: A variety of computed time dependent galactic flow models are compared at time $t_{n}=13$ Gyrs with the observed gas density (top row) and temperature (middle row) of NGC 4472 taken from Figure 2. The bottom row shows the computed hot gas iron abundance compared with the observations of Buote (2000c). First column: A plot of the profiles at $t_{n}$ for an approximate model of NGC 4472 assuming an isolated galaxy (solid lines); this "reference" flow is based on an assumed parameter set as explained in the text. A similar galactic flow but including initial circumgalactic gas ("cgg") is also shown (dashed lines). Second column: A model similar to the reference flow but with ad hoc spatially distributed cooling with dropout parameter $q=1$. Third column: Two flows similar to the reference flow (for which $\mathrm{SNu}\left(t_{n}\right)=0.06$ ) but with additional heating by Type Ia supernovae: $\mathrm{SNu}\left(t_{n}\right)=0.18$ (solid lines) and $\mathrm{SNu}\left(t_{n}\right)=0.25$ (dashed lines). Fourth column: Three galactic flows similar to the reference flow but calculated with different past Type Ia supernova rates $\mathrm{SNu}(t) \propto t^{-s}: s=0$ (solid lines), 1.5 (dashed lines) and 2 (dotted lines). In the reference model $s=1.3$. 
beyond the optical galaxy $(r \gtrsim 10 \mathrm{kpc}$ ) is increased to fit the data. (2) Since the virial temperature of the NFW group halo exceeds that of the central galaxy, the gas temperature of the circumgalactic gas is higher. As hot circumgalactic gas flows inward, it is cooled by radiation losses and by mixing with stellar ejecta at characteristic temperature $\sim T_{*} \sim 10^{7} \mathrm{~K}$, naturally creating the observed positive temperature gradient within $\sim 50 \mathrm{kpc}$. All known E galaxy temperature profiles are positive (Figure $2 \mathrm{~b}$ ) within several $R_{e}$ and must therefore contain hot circumstellar gas, but when those with very small X-ray images are observed (e.g. NGC 4374: Mathews \& Brighenti 1998), it is possible that $d T / d r$ will be negative as in the "isolated" reference model. (3) If the iron abundance in the inflowing circumgalactic gas is $z_{F e} / z_{F e, \odot} \sim 0.3-0.4$, typical of external regions in groups and clusters, then as the inflowing gas mixes with supernova ejecta, the mean abundance $z_{F e} / z_{F e, \odot} \sim 1-2$ at $r \sim 10 \mathrm{kpc}$ is closer to observed values. The beneficial effect of these improvements, taken together, provide support for subsonic inflow as in classical cooling flow theory.

Central Mass Difficulties: Our reference solution has serious problems near the origin. The amount of gas that has cooled there by time $t_{n}, M_{\text {cold }}=37 \times 10^{9}$ $M_{\odot}$, far exceeds the mass $M_{b h} \sim 0.56-2.6 \times 10^{9} M_{\odot}$ of the central black hole observed in NGC 4472 (e.g. Magorrian et al. 1998; Merritt \& Ferrarese 2001) and would cause the central stellar velocity dispersion to exceed the observed value. In standard cooling flows in luminous E galaxies, with or without circumgalactic gas, the mass of cooled gas, $M_{\text {cold }}$, is several times greater than the total mass of hot gas at $t_{n}$. Although most of the cooled mass $M_{\text {cold }}$ is formed at early times $\left(\alpha_{*} \propto t^{-1.3}\right)$ when our understanding of galactic evolution is uncertain, at the current cooling rate for the reference flow, $\dot{M} \approx 1.1 M_{\odot} \mathrm{yr}^{-1}, M_{\text {cold }}$ and $M_{b h}$ become equal after only $\sim 2$ Gyrs. $M_{\text {cold }}$ can also be reduced by supernova driven galactic winds at early times; this may occur even before the E galaxy formed by mergers. If the reference model is begun gas-free at a much later time $t_{\text {in }}=5$ Gyrs (redshift $z \sim 1.25$ for $H_{0}=70, \Omega_{m}=0.3$ and $\Omega_{\Lambda}=0.7$ ), then $M_{\text {cold }}=7.7 \times 10^{9} M_{\odot}$ is reduced but still exceeds $M_{b h}$ - the problem of excessive $M_{\text {cool }}$ does not go away easily.

Compact, luminous X-ray emission is expected as hot interstellar gas flows toward the central supermassive black holes in E galaxies (Fabian \& Canizares 1988). In our reference flow, even if the potential energy of the black hole is ignored, the X-ray luminosity of gas cooling by thermal emission at the very center of the flow, $L_{x, b o l}\left(r=0, t_{n}\right) \approx\left(5 k T / 2 \mu m_{p}\right) \dot{M}(0) \approx 10^{41} T_{7}\left(\dot{M} / M_{\odot} \mathrm{yr}\right) \sim 10^{41}$ ergs $\mathrm{s}^{-1}$, is comparable to $L_{x, b o l}$ from the rest of the flow, in flagrant violation of observations. Attempts to detect compact X-ray sources in giant $\mathrm{E}$ galaxies have been remarkably unsuccessful (e.g. Fabian \& Rees 1995; Reynolds et al. 1996; Di Matteo et al. 2000; Roberts \& Warwick 2000; Loewenstein et al. 2001; Sulkanen \& Bregman 2001). This emission shortfall is usually expressed in terms of the luminosity $L \approx \eta \dot{M} c^{2}$ of spherical Bondi accretion onto a mass point, $\dot{M} \approx 4 \pi \rho_{\infty} c_{\infty}^{-3}\left(G M_{b h}\right)^{2}$, where $\rho_{\infty}$ is the density of distant gas at rest and $c_{\infty} \approx 370 T_{7}^{1 / 2} \mathrm{~km} \mathrm{~s}^{-1}$ is the isothermal sound speed in this gas. If energy is produced with efficiency $\eta=0.1$, the expected luminosity $L \sim \eta \dot{M} c^{2} \sim 6 \times 10^{44}(\eta / 0.1) n_{e} T_{7}^{-3 / 2}\left(M_{b h} / 10^{9} M_{\odot}\right)^{2}$, is similar to a quasar. Central X-ray emission from Chandra observations of NGC 6166 (Di Matteo et al. 2001) and M87 (Di Matteo et al. 2003), where nuclear X-ray sources are observed, indicate $\eta \sim 10^{-4}$. This low efficiency is within the radiation-reducing 
capability of advection dominated accretion flows (ADAFs) (Rees et al. 1982; Narayan \& Yi 1995; Abramowicz et al. 1995). The $\dot{M}$ in M87 and NGC 6166 may also be reduced below the Bondi rate by occasional AGN heating. In addition, some of the accreting mass and energy may be redirected to kinetic flow along a jet (Blandford \& Begelman 1999) which for M87 is $\sim 10^{44} \mathrm{erg} \mathrm{s}^{-1}$. Recently Loewenstein et al (2001) examined Chandra images of several bright E galaxies (NGC 1399, NGC 4472, and NGC 4636) and found no evidence of compact nuclear X-ray emission in the galactic cores, indicating $\eta \lesssim 10^{-5}$. Either the radiative efficiency is incredibly low or gas is not arriving at the black hole in Bondi flow. Perhaps the gas is outflowing in this region or heated in some way by the black hole; these possibilities would be compatible with the flat density gradient observed in the central few kpc of M87 (Di Matteo et al. 2002) that is difficult to produce with inflowing or static solutions.

Mass Dropout: The traditional device to avoid huge central masses of cooled gas and central inflows at $t_{n}$ has been to assume that the cooling is somehow spread over a large range of radius. To accomplish this, an ad hoc "dropout" term is added to the right hand side of Equation $(3),-q \rho / t_{c o o l}$, where $q$ is a dimensionless parameter (e.g. Fabian, Nulsen \& Canizares 1984a; White \& Sarazin 1987; Sarazin \& Ashe 1989; Kritsuk 1992). This term is designed to force the gas to cool at any radius in proportion to the local gas density divided by the local cooling time at constant pressure, $t_{\text {cool }}=5 m_{p} k T / 2 \mu \rho \Lambda$. For constant $q$ the rate of cooling dropout, $q \rho / t_{\text {cool }} \propto n_{e}^{2}$, is concentrated toward the galactic center, but $q$ can also be assumed to vary with galactic radius. Sarazin \& Ashe (1989) showed that models with $q \approx 1$ fit the X-ray data reasonably well. It is interesting to estimate the value of $q$ that just balances mass loss from stars in Equation (3), $q=\left(t_{\text {cool }} / \rho\right) \alpha_{*} \rho_{*} \approx 0.4$ where $T=10^{7} \mathrm{~K}$ is assumed and we use $\rho_{*} \approx 8.54 \times 10^{-20} n_{e}^{2}$ from Figure $2 \mathrm{a}$. Therefore, in evolutionary models with uniform $q \sim 1$ gas is removed from the flow at about the same rate that it is supplied by stars. The flow does not shut down if $q>1$, however, since the gas density decreases and $q \rho / t_{\text {cool }} \propto \rho^{2}$ becomes less effective; the total mass of cooled gas is quite independent of $q$.

In column 2 of Figure 5 we plot the density and temperature using reference model parameters but including the dropout term in Equation (3) with $q=1$. One of the historical motivations for dropout was to avoid the central rise in gas density in cooling flow models, but in our experience dropout does not completely solve this difficulty as seen in Figure 5. In this dropout solution the mass of cooled gas, $M_{\text {cold }}=3.8 \times 10^{10} M_{\odot}$, is almost the same as in the reference solution, but only a small fraction cools at the origin. In flows with mass dropout the gas is multiphase everywhere, i.e. some gas cools in pressure equilibrium at every radius and passes through a continuum of higher densities and lower temperatures. The additional emission from these cooling regions, if they exist, is substantial and must be added to the emission of the smooth background gas that radiates in the normal way. Consequently, the observed or apparent gas density is higher and the temperature lower than that of the smooth background. The apparent density profile $n_{e}(r)$ in column 2 of Figure 5 agrees much better with the data for NGC 4472 than the reference model $(q=0)$ in column 1. Adding circumgalactic gas would improve the agreement further.

If the cooled gas forms into a spatially extended population of optically dark (dwarf) stars, as often assumed, then the stellar mass to light ratio would vary with galactic radius. In some cases this dark mass can thicken and distort the 
fundamental plane beyond observed limits (Mathews \& Brighenti 2000). But in the high pressure environment of galactic flows, stable Bonner-Ebert spheres at $10^{4} \mathrm{~K}$ have masses $\lesssim 2 M_{\odot}$, and this may also be the maximum mass of any stars that form (Mathews \& Brighenti 1999a); because these stars are optically luminous, their influence on the fundamental plane is lessened. Young stars in this mass range could explain the high stellar $\mathrm{H} \beta$ features that are commonly observed in giant E galaxies (Mathews \& Brighenti 1999b; Terlevich \& Forbes 2002).

Another historic difficulty with the dropout hypothesis is that infinitesimal perturbations in the gas density do not develop into full blown thermal instabilities (e.g. Balbus 1991). Loewenstein (1989) showed that small (coherent!) density perturbations oscillate radially in the nearly static hot gas atmosphere with very little overdensity on average and do not cool appreciably faster than the ambient undisturbed gas. Computational studies of the gas dynamics of initially overdense regions in cooling flows (Hattori \& Habe 1990; Yoshida, Habe \& Hattori 1991; Malagoli, Rosner \& Fryxell 1990; Reale et al. 1991; Hattori, Yoshida \& Habe 1995) indicate that runaway thermal instabilities are not expected unless the initial perturbation amplitude is very large, $\delta \rho / \rho \gtrsim 1$. However, in recent $2 \mathrm{D}$ calculations of AGN heated flows spatially distributed cooling appeared spontaneously near the outer boundary of the convective region (Kritsuk, Plewa \& Müller 2001) and also in non-linear compressions in convective regions (Brighenti \& Mathews 2002b).

Nevertheless, intermediate (multiphase) temperatures, an essential outcome of radiative cooling and mass dropout, are not supported by XMM X-ray spectra of galactic scale flows (NGC 4636: Xu et al. 2002; NGC 5044: Buote et al. 2003a; NGC 1399: Buote 2002; M87: Molendi \& Pizzolato 2001). Likewise, in cluster scale flows there is no evidence for gas cooling below $\sim 1-2 \mathrm{keV}$ (Peterson et al. 2001; Tamura et al. 2001; Kaastra et al. 2001; Molendi \& Pizzolato 2001; Böhringer et al. 2002; Matsushita et al. 2002). These astonishing null results have led to many speculations, discussed below, but at present no single explanation is generally accepted.

Transition to Winds: Clearly, it could be helpful if the gas flowed out rather than in, but what additional heating is required to drive a wind at time $t_{n}$ ? To answer this question, we heated the gas by increasing the Type Ia supernova rate in NGC 4472 above the reference value $\mathrm{SNu}\left(t_{n}\right)=0.06 \mathrm{SNu}$ and repeated the calculation with all other parameters (including $s$ ) unchanged. The transition to a wind is abrupt. As seen in column 3 of Figure 5 , for $\mathrm{SNu}\left(t_{n}\right)=0.18$ the temperature and density profiles at $t_{n}$ are almost identical to the reference solution, although $M_{\text {cold }}=2.5 \times 10^{10} M_{\odot}, M_{h o t}=2.4 \times 10^{10} M_{\odot}$ and $L_{x, b o l}=$ $1.0 \times 10^{42} \mathrm{erg} \mathrm{s}^{-1}$ are all slightly lower due to outflows at early times. At time $t_{n}$ the gas is flowing inward at all radii. However, a further small increase to $\mathrm{SNu}\left(t_{n}\right)=0.25$ produces a strong global wind at $t_{n}$ with very low gas density at all radii and $L_{x, b o l}$ drops to $1.9 \times 10^{41} \mathrm{erg} \mathrm{s}^{-1}$. No known galaxy has density, temperature and abundance profiles like those for the $\mathrm{SNu}\left(t_{n}\right)=0.25$ solution in Figure 5. Outflows generally require finely-tuned heating. More realistic galactic flows with additional circumgalactic gas require a much larger $\mathrm{SNu}\left(t_{n}\right)$ to drive an outflow by $t_{n}$. Outflows may be common in low luminosity ellipticals, $L_{b} \lesssim$ $L_{B, \text { crit }}$, and spiral bulges where the hot gas is difficult to observe. If the reference Type Ia rate $\mathrm{SNu}\left(t_{n}\right)=0.06 \mathrm{SNu}$ is applied to elliptical galaxies that are $\lesssim 0.3$ as luminous as NGC 4472 , outflows at $t_{n}$ are easy to generate. 
Past Supernova Rate: Next we describe several flows at $t_{n}$ for a variety of past supernova rates $\mathrm{SNu}(t) \propto t^{-s}$, by varying the index $s$ from 0 to 2 , keeping $\mathrm{SNu}\left(t_{n}\right)=0.06$ fixed. In row 3 , column 4 of Figure 5 we show the current $\left(t=t_{n}\right)$ hot gas iron abundance profiles for flows with $s=0,1.5$ and 2. The temperature and density profiles at $t_{n}$ change little over this range of $s . M_{\text {cold }}$ decreases by about 4.5 as $s$ increases from 0 to 2 because of supernova-driven outflows at early times. $L_{x, b o l}\left(t_{n}\right)$ only decreases by 40 percent as $s$ varies from 0 to 2 . Recall that $s>1.3(s<1.3)$ is a necessary condition for supernova driven winds to occur at early (late) times.

Ciotti et al. (1991) considered evolutionary models with $s=1.5$ and $\mathrm{SNu}\left(t_{n}\right)=$ $0.11-0.22 \mathrm{SNu}$, so that outflows and winds occur at early times, thereby reducing $M_{\text {cool }}\left(t_{n}\right)$. They also assumed that the fraction of galactic mass in dark halos varies among elliptical galaxies with similar $L_{B}$. As a result, the model ellipticals described by Ciotti et al. are at the present time in different phases of a transition from outflow (low $L_{x, b o l} / L_{B}$ ) to cooling inflow (high $L_{x, b o l} / L_{B}$ ) and they interpret this as an explanation for the large scatter in the $L_{x}, L_{B}$ plot. However, for the same range of supernova parameters, the variations in $L_{x} / L_{B}$ would be greatly reduced if circumgalactic gas had been included. Furthermore, the iron abundance at $t_{n}$ is strongly linked to the past supernova rate (Loewenstein \& Mathews 1991; Brighenti \& Mathews 1999b). Type Ia rates required to drive winds at early times deposit too much iron in the hot gas by time $t_{n}$. The iron abundance for our $s=1.5$ galaxy in Figure 5 is $\sim 3.5$ solar throughout the hot gas. This high abundance cannot be satisfactorily reduced by mixing with inflowing circumgalactic gas (column 1, Figure 5). We conclude that the transient evolution from supernova-driven winds to inflows is unlikely to explain the large scatter in the $L_{x}, L_{B}$ plot for massive ellipticals $\left(L_{B} \gtrsim 3 \times 10^{10} L_{B, \odot}\right)$.

Additional Parameters: Finally, we note several additional parameters that have some influence on the solutions at time $t_{n}=13$ Gyrs. Varying the (uniform) stellar temperature $T_{*}$ from $6 \times 10^{6} \mathrm{~K}$ (the reference flow value) to $2 \times 10^{6} \mathrm{~K}$ or $18 \times 10^{6} \mathrm{~K}$ (but keeping the stellar mass fixed) leaves $n_{e}(r)$ and $T(r)$ essentially unchanged. Matsushita (2001) argues that $L_{x}$ and the hot gas temperature in E galaxies without circumgalactic gas can be explained by kinematical heating of the gas by stellar mass loss. However, the kinematic temperature $T_{*}$ of mass lost from orbiting stars is essentially the same as the hot gas temperature, i.e., both are determined by the same gravitational potential. Altering the radiative cooling coefficient $\Lambda(T)$ by factors 3 or $1 / 3$ has no appreciable effect on $T(r)$ but the gas density increases slightly with decreasing $\Lambda$. If $\Lambda=0$, as if some source of heating perfectly balances radiative cooling at every radius, then there is no radial flow and no gas cools at any radius. But in this strange solution the gas density becomes very large and the density gradient steepens $\left(\rho \propto \rho_{*}\right)$ because a larger pressure gradient is required to support the denser, nearly static atmosphere. Decreasing the mass of the NFW halo from the reference value $M_{h}=4 \times 10^{13} M_{\odot}$ to $M_{h}=5 \times 10^{12} M_{\odot}$ leaves $n_{e}(r)$ essentially unchanged but $T(r)$ is $\sim 20$ percent lower at $r=10 \mathrm{kpc}$. For $M_{h}=10^{12} M_{\odot}$, however, a mild galactic wind sets in at late times and the density and temperature are both significantly lower. In this last solution $L_{x, b o l}$ is $\sim 30$ times lower than the reference value and comparable to the luminosity of discrete stellar X-ray sources in NGC 4472, $L_{x, *} \approx 3 \times 10^{40} \mathrm{erg} \mathrm{s}^{-1}$ (Figure 1). It is also interesting to vary the stellar mass loss rate $\alpha_{*}$, simulating a situation in which not all of the gas ejected from stars goes into the hot phase. When $\alpha_{*}\left(t_{n}\right)$ is reduced by 2 , holding $\alpha_{s n}$ at 
its reference value, the total mass of cooled gas $M_{\text {cold }}$ and $L_{x}$ are both lowered by 2. The gas temperature within $\sim 40 \mathrm{kpc}$ is lower by $\sim 1.4$, but the total mass of hot gas $M_{h o t}=1.1 \times 10^{10} M_{\odot}$ is almost unchanged. If $\alpha_{*}\left(t_{n}\right)$ is lowered further, a wind develops by time $t_{n}$.

\section{THE COOLING PARADOX - DOES THE GAS COOL?}

The cooling paradox refers to the remarkable discovery that the hot gas radiates but does not seem to cool. Many who have been closely involved with so-called "cooling flows" now doubt that they are cooling and some question whether they are flowing. Current attempts to resolve this paradox are generally taking two approaches: (1) the gas is cooling, but for some reason it evades detection and/or (2) the gas is being heated in some way so that very little gas cools. In this section we briefly review the evidence supporting the first approach, and heating is discussed in the following section.

\subsection{Spectroscopic and Morphological Cooling Rates}

Most of the evidence that cooling is incomplete or absent comes from XMM RGS (Reflection Grating Spectrometer) spectra of cluster scale flows (Abell 1835: Peterson et al. 2001; Abell 1795: Tamura et al. 2001; Abell S1101: Kaastra et al. 2001). In general the older data from ROSAT and ASCA could not easily distinguish between flows with a radially varying single temperature (i.e. single phase) and truly multiphase flows in which a range of temperatures is also present at every radius. For Abell 1835, a massive cluster with $T_{\text {vir }} \approx 9 \mathrm{keV}$, Allen et al. (1996) estimated a large mass deposition rate, $\dot{M} \approx 2000 M_{\odot} \mathrm{yr}^{-1}$, using an $\mathrm{X}$-ray image deprojection procedure based on a steady state cooling flow model. This cooling rate has now been adjusted downward to $\dot{M}<200 M_{\odot} \mathrm{yr}^{-1}$ as a result of the XMM RGS spectrum of Abell 1835 that shows no emission lines from ions at temperatures $T \lesssim T_{\text {vir }} / 3$ (Peterson et al. 2001). Similar drastic reductions were made to morphological $\dot{M}$ estimates for other clusters, although the RGS only views the central 1' of each cluster, a radius of 30". In these clusters, the gas temperature drops from $T_{\text {vir }}$ at large radii to about $\sim T_{\text {vir }} / 3$ near the center, similar to Figure 2b, with little or no indication of multiphase (cooling) gas at lower temperatures, $k T \lesssim 1-2 \mathrm{keV}$.

At present the XMM and Chandra data for galaxy/group flows are limited, but the spectroscopic $\dot{M}$ also seems to be significantly lower than the morphological $\dot{M}$, as in cluster flows. XMM RGS observations of NGC 4636 by Xu et al. (2002) indicate that the gas temperature decreases from $8.1 \times 10^{6} \mathrm{~K}$ at $3 \mathrm{kpc}$ to $6.4 \times 10^{6}$ $\mathrm{K}$ at the center, but there is little or no spectroscopic evidence for gas emitting at temperatures $\lesssim 6 \times 10^{6} \mathrm{~K}$. The OVII line at $0.574 \mathrm{keV}$ that emits near $2.5 \times 10^{6} \mathrm{~K}$ is not detected. Xu et al. determine $\dot{M}<0.3 M_{\odot} \mathrm{yr}^{-1}$ within $r \sim 2.5 \mathrm{kpc}$. This is less than the total stellar mass loss rate expected in NGC 4636, $\alpha M_{* t} \approx 0.5 M_{\odot}$

$\mathrm{yr}^{-1}$, but most of the gas in NGC 4636 is circumgalactic so the mass cooling rate expected in a traditional cooling flow model would be larger: $\dot{M} \approx 1-2 M_{\odot} \mathrm{yr}^{-1}$ at distance $d=17 \mathrm{Mpc}$ (e.g. Bertin \& Toniazzo 1995). However, Bregman, Miller \& Irwin (2001) have observed NGC 4636 with the Far Ultraviolet Spectroscopic

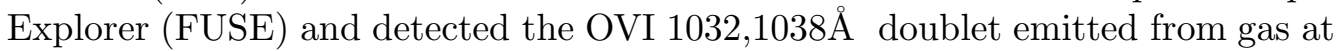
$T \sim 3 \times 10^{5} \mathrm{~K}$. It is generally assumed that this emission arises from gas that is cooling, not from the stellar ejecta as it is being heated to the hot gas phase. The 
heating process, possibly involving shocks and rapid thermal mixing, is likely to be faster and more efficient than collisional excitation of emission lines during cooling (Fabian, Canizares, \& Böhringer 1994). The FUSE observations indicate a cooling rate of only $\dot{M} \approx 0.17 \pm 0.02 M_{\odot} \mathrm{yr}^{-1}$ for $d=17 \mathrm{Mpc}$. Both XMM RGS and FUSE observations are consistent with a cooling rate that is $\sim 5$ times less than predicted from cooling flow models. However, FUSE has only a 30" $(2.5 \mathrm{kpc})$ square aperture containing $\sim 0.083$ of the total X-ray luminosity. If the cooling were spatially distributed in proportion to the X-ray surface brightness, as reckoned by Bregman et al., the total cooling rate in NGC 4636 would be $\sim 0.17 / 0.083 \sim 2 M_{\odot} \mathrm{yr}^{-1}$, which is consistent with traditional cooling flow models. The OVI line width observed with FUSE is $44 \mathrm{~km} \mathrm{~s}^{-1}$. However, if the OVI emission shares the same kinematic widths $\sim 150 \mathrm{~km} \mathrm{~s}^{-1}$ as the $\mathrm{H} \alpha+$ [NII] lines observed in the central 15" of NGC 4636 by Caon et al. (2000), the additional flux in the broad wings might not have been detected by FUSE.

Spectroscopic X-ray and FUSE cooling rates for M87 and NGC 1399, respectively centered in the Virgo and Fornax clusters, are also much less than previously expected morphological rates. XMM-EPIC observations of M87 indicate $\dot{M} \lesssim 0.5 M_{\odot} \mathrm{yr}^{-1}$ (Molendi \& Pizzolato 2001; Matsushita et al. 2002), much less than earlier predictions from X-ray images: $\sim 40 M_{\odot} \mathrm{yr}^{-1}$ (Peres et al. 1998) and $\sim 10 M_{\odot} \mathrm{yr}^{-1}$ (Stewart et al. 1984; Fabian et al. 1984b). In NGC 1399 Buote (2002) found that the XMM spectrum within $\sim 20 \mathrm{kpc}$ is best fit with a two temperature emission model; spectral models with a single temperature or a continuum of temperatures (as in a cooling flow) give less satisfactory fits. Buote identifies the hotter phase $(1.5-1.8 \mathrm{keV})$ with gas from the Fornax cluster and the colder phase $(1.0-1.3 \mathrm{keV})$ with gas ejected from stars in NGC 1399, but there is no evidence that the cool phase cools to even lower temperatures. This is supported by FUSE observations of NGC 1399 by Brown et al. (2002) who find $\dot{M}<0.2 M_{\odot} \mathrm{yr}^{-1}$ within a $3 \times 3 \mathrm{kpc}$ region at the center of NGC $1399-$ this is much less than the total cooling rate of $\sim 2 M_{\odot} \mathrm{yr}^{-1}$ inferred from the X-ray image using cooling flow models (Bertin \& Toniazzo 1995; Rangarajan et al. 1995). Perhaps the cooling is intermittent.

\subsection{Warm and Cold Gas at $\mathrm{T}<10^{5} \mathrm{~K}$}

In view of the failure to detect significant cooling at X-ray temperatures, it is remarkable that faint, diffuse, warm gas at $T \sim 10^{4} \mathrm{~K}(\mathrm{H} \alpha+[\mathrm{NII}])$ is almost universally observed near the centers of groups and clusters formerly identified as cooling flows. Although the mass of warm gas $\left(10^{4}-10^{5} M_{\odot}\right)$ is very much less than the mass that would have cooled in the cooling flow scenario, it could represent an important short-lived phase in the cooling process. The density of this warm gas, estimated from the [S II] 6716/6731 ratio, is $\sim 100 \mathrm{~cm}^{-3}$ (Heckman et al 1989; Donahue \& Voit 1997) compatible with pressure equilibrium with the hot gas within $\sim 1 \mathrm{kpc}$ of the galactic centers. The warm gas can be ionized by galactic starlight from old, hot post-AGB stars (Binette et al 1994) or by UV from locally cooling gas and thermal conduction (Donahue \& Voit 1991; 1997). The total luminosity in $\mathrm{H} \alpha+[\mathrm{NII}]$ emission is $\sim 1-10 \times 10^{39} \mathrm{erg} \mathrm{s}^{-1}$. In $\mathrm{E}$ galaxies that are strong radio sources most of the line emission is concentrated within $\sim 1$ arcsecond of the center (Kleijn et al. 2002), but it is unclear if this is true in general.

Recent surveys of $\mathrm{H} \alpha+[\mathrm{NII}]$ images and kinematics in elliptical galaxies 
(Shields 1991; Buson et al. 1993; Goudfrooij et al. 1994; Macchetto et al. 1996; Caon, Macchetto \& Pastoriza 2001) reveal that the images are rarely as smooth and regular as the stellar isophotes. The spatial irregularities in the $\mathrm{H} \alpha+[\mathrm{NII}]$ images appear to be compatible with the random $\mathrm{H} \alpha+[\mathrm{NII}]$ velocities $\sim \pm 150$ $\mathrm{km} \mathrm{s}^{-1}$ observed (Zeilinger et al. 1996; Caon et al. 2000). The warm gas clearly does not track the smooth rotation or dispersion kinematics of the stars but instead may approximately follow the motion of the hot gas. If so, the hot gas would be subsonically turbulent with energy on spatial scales $(\sim 0.5 \mathrm{kpc})$ too large to be produced by Type Ia supernovae.

A small number of elliptical galaxies have detectable neutral or molecular gas. Surveys (Knapp, Turner \& Cunniffe 1985; Huchtmeier 1994; Huchtmeier, Sage \& Henkel 1995) detect HI emission in $\lesssim 15$ percent of E galaxies, but in some cases this may include emission from nearby late type galaxies within the beam. HI disks are more common in low luminosity E galaxies that do not have observable X-ray emission from hot gas (Sadler, et al. 2000; Oosterloo et al. 2002). Some of the more spectacular examples, in which the HI gas probably results from a recent merger, have blue colors like spiral galaxies. Similarly, Wiklind, Combes \& Henkel (1995), Knapp \& Rupen (1996) and Young (2002) detect CO emission from a relatively small number of E galaxies selected because of their large far infrared fluxes. The molecular gas is often rotating in large $(1-10 \mathrm{kpc})$ disks of mass $10^{7}-10^{9} M_{\odot}$. In some cases the high specific angular momentum of the molecular gas (or its sign) requires an external origin. Using spectroscopic stellar ages, Georgakakis et al. (2001) have shown that the mass of neutral and molecular gas decreases with time (by star formation?) since the last merger event.

\subsection{Dust in Elliptical Galaxies}

About 80 percent of elliptical galaxies have dust clouds, lanes or disks within $\sim 1 \mathrm{kpc}$ of the center (van Dokkum \& Franx 1995; Martel et al. 2000; Colbert, Mulchaey \& Zabludoff 2001; Tran et al. 2001; Kleijn et al. 2002) with associated gas masses $10^{4}-10^{7} M_{\odot}$. Some of the $\mathrm{H} \alpha$-emitting gas is undoubtedly related to the photoionized boundaries of these dusty clouds (Goudfrooij et al. 1994; Ferrari et al. 1999). One might expect the dusty cores having an irregular (non-disk) appearance to be related to recent AGN outbursts, but the evidence for this is not yet compelling (e.g. Tomita et al. 2000; Krajnovic \& Jaffe 2002). If AGN energy outbursts are common in E galaxies, it is remarkable that these rather fragile central dusty regions have survived. IRAS luminosities, detected in $\sim 50$ percent of ellipticals, indicate that there is much more dust in E galaxies than that responsible for the optical extinction in the central clouds (Goudfrooij \& de

Jong 1995; Bregman et al. 1998: Merluzzi 1998; Ferrari et al. 2002). The ratio of $100 \mu \mathrm{m}$ to optical B-band fluxes is noticeably higher for $\mathrm{cD}$ galaxies (Bregman, McNamara \& O'Connell 1990). Knapp, Gunn and Wynn-Williams (1992) have shown that the mid-infrared $\sim 12 \mu \mathrm{m}$ radiation has an $r^{1 / 4}$ distribution like the galactic stars, consistent with circumstellar dust associated with stellar mass loss. Recent midIR ISO spectra show the $9.7 \mu \mathrm{m}$ feature characteristic of circumstellar silicate grains expected in low mass AGB stars (Athey et al. 2002), this is proof that galactic stars are ejecting $\alpha$-elements. According to Athey et al., the total midIR emission is consistent with typical stellar mass loss rates $\sim 1 M_{\odot} \mathrm{yr}^{-1}$ in luminous E galaxies, although $\dot{M}$ may not scale with $L_{B}$. 


\subsection{Origin of Warm Gas, Cold Gas and Dust}

The diffuse warm $\left(\sim 10^{4} \mathrm{~K}\right)$ gas that emits optical emission lines gas could have been recently ejected from stars, cooled from the hot phase, acquired in a recent galactic merger, or accreted from local gas clouds similar to the high velocity clouds that fall toward the Milky Way. Interesting morphological similarities between the $\mathrm{H} \alpha+[\mathrm{NII}]$ and X-ray images have been reported in some E galaxies (Trinchieri \& di Serego Alighieri 1991; Trinchieri, Noris, \& di Serego Alighieri 1997; Trinchieri \& Goudfrooij 2002), suggesting a generic relationship. Optical line emission traces (part of) the perimeters of X-ray cavities in 3C317/Abell 2052 (Blanton et al. 2001b) and Perseus/NGC 1275 (McNamara, O'Connell \& Sarazin 1996), but apparently does not fill in the cavities. This may be significant. Since the cavity rims show little heating due to strong shocks, the pressure inside the cavity must be similar to that in the rims. If so, recent stellar ejecta or warm gas introduced by a merger should be just as luminous inside the cavities, but evidently this is not observed. Either the evaporation-heating time is shorter inside the cavities or the $\mathrm{H} \alpha$-emitting gas may have cooled from the hot phase.

The total $\mathrm{H} \alpha$ luminosity among luminous E galaxies, $L_{H \alpha} \approx 10^{39.5 \pm 1.0} \mathrm{ergs}$ $\mathrm{s}^{-1}$ (Goudfrooij et al. 1994), holds for E galaxies for which $L_{x}$ varies by $\sim 10^{3}$. This uniformity and upper bound $\left(L_{H \alpha} \lesssim 10^{40.5} \mathrm{ergs} \mathrm{s}^{-1}\right)$ on the line emission may reflect the similarity of central hot gas pressure among massive E galaxies, again suggesting (but not proving) an internal origin for the warm gas. Mergers with gas-rich dwarfs might produce a wider spread in $L_{H \alpha}$. Evidently all nearby X-ray bright E galaxies contain diffuse $\mathrm{H} \alpha+[\mathrm{NII}]$ emission. It seems unlikely that mergers with gas-rich galaxies would be equally common for E galaxies in small groups and those in richer clusters. The tendency for some of the diffuse $\mathrm{H} \alpha$ $+[\mathrm{NII}]$ images to approximately follow the stellar isophotes (Caon et al. 2000) also suggests an internal source for the warm gas, but the exceptions (e.g. NGC 5044) could arise from a merger or from recent AGN energy release.

The strange velocity patterns in the $\mathrm{H} \alpha+[\mathrm{NII}]$ emission (e.g. Caon et al. 2001) may also constrain the origin of this warm gas. The total stellar mass loss rate and hot gas cooling rate in classical "cooling flows" are both $\sim 1 M_{\odot}$ $\mathrm{yr}^{-1}$ in large E galaxies. If this warm gas has a stellar origin, its lifetime must be short, $t_{\text {warm }} \lesssim 10^{5}$ yrs, to keep $L_{H \alpha}$ within observed limits (Mathews \& Brighenti 1999a). The velocity distribution of the warm gas is very different from that of the stars. But the warm gas clouds are $T_{\text {hot }} / T_{\text {warm }} \sim 1000$ times denser than the hot gas, so they cannot be be drag-accelerated to the local hot gas velocity in time $t_{\text {warm }}$. Therefore the $\mathrm{H} \alpha+[\mathrm{NII}]$ emitting gas cannot be swept to the rims of the X-ray cavities by the motion of the hot gas. The chaotic velocity structure of the warm gas could be understood either as the undissipated motions of a very recent merger, or (more likely ?) as turbulent motions in the hot gas from which it cooled. The obvious difficulty with this second possibility is that XMM observations so far provide little or no evidence for cooling.

It is often claimed that most or all of the cold gas and dust in E galaxies is a result of mergers with gas-rich galaxies (e.g. Sparks et al. 1989; Sparks 1992; de Jong et al. 1990; Zeilinger et al. 1996; Caon et al. 2000; Trinchieri \& Goudfrooij 2002). If the warm gas has a finite lifetime, an ongoing supply of new gas is required. However, there are relatively few reported cases of gas-rich dwarf galaxies currently merging with giant E galaxies although the optical line emission would make such merging galaxies easy to find. Moreover, most of the gas in 
merging galaxies could be lost by ram pressure stripping during the $10^{8}-10^{9}$ yrs as they orbit toward the center of the giant E galaxy. Undoubtedly, however, mergers do occur. A sure signature for mergers are the counter-rotating warm gas clouds found in some E galaxies by Zeilinger et al. (1996) and Caon et al. (2000). Further detailed observations of warm gas distributions and kinematics in normal $\mathrm{E}$ galaxies are needed to better constrain its origin.

\subsection{Hiding the Cooling Gas}

The spectral contributions of cooling gas can be reduced if X-ray emission from the cooling gas is absorbed or if the cooling is more rapid than normal radiative cooling (Peterson et al. 2001; Fabian et al. 2001).

Intrinsic X-ray absorption has been invoked to interpret ROSAT and ASCA observations of cluster cooling flows (e.g. Allen 2000; Allen \& Fabian 1997; Allen et al. 2001). Column densities $N$ of a few $10^{21} \mathrm{~cm}^{2}$ are sufficient to absorb 1 $\mathrm{keV} \mathrm{X}$-rays provided the absorbing gas is reasonably cold, $T \lesssim 10^{6} \mathrm{~K}$. Because X-ray absorption varies rapidly with energy, $\sigma(E) \propto E^{-3}$, it can reduce the low temperature contributions to the $\sim 1 \mathrm{keV}$ FeL iron line complex, simulating the absence of cooler gas (Böhringer et al. 2002). At ASCA resolution spectral models with conventional cooling flows are possible if the low temperature gas is largely hidden by intrinsic X-ray absorption. Buote (2000b,d; 2001) and Allen et al. (2001) reported evidence for an absorbing oxygen edge (at $\sim 0.5$ $\mathrm{keV}$ ) and intrinsic absorbing columns $N \sim 10^{21} \mathrm{~cm}^{2}$ within $\sim 5-10 \mathrm{kpc}$ in several bright E galaxies such as NGC 5044 and NGC 1399. But the mass of absorbing gas required to occult the central regions of these galactic flows is large, $M_{a b s} \approx(4 / 3) \pi r^{3} n m_{p} \sim 3 \times 10^{7} r_{k p c}^{2} N_{21} M_{\odot}$ with unit filling factor. It is unclear how this (necessarily colder) gas would be supported in the galactic gravity field and how it would escape detection at other frequencies (Voit \& Donahue 1995). Absorbing gas with $N \gtrsim 10^{20} \mathrm{~cm}^{2}$ would produce observable reddening of the background stars and large far-infrared luminosities unless the gas is dust-free. Resonant X-ray lines emitted from ions expected at low-temperatures can in principle be scattered and then absorbed by the continuous X-ray opacity (Gil'fanov et al. 1987), but the line-center optical depths are small and not all of the missing lines in XMM spectra are resonance transitions (Peterson et al. 2001). XMM and Chandra spectra of the non-thermal nucleus and jet of M87 and the nucleus of NGC 1275 fail to show intrinsic X-ray absorption at the level required to mask cooling in the thermal gas (Böhringer et al. 2001). Perhaps the ideal X-ray absorption model would be one in which the cooling regions are themselves optically thick at $\lesssim 1 \mathrm{keV}$, but our attempts to achieve this have been unsuccessful. Overall, the prospects for intrinsic X-ray absorption are not particularly encouraging.

If the cooling rate at $k T \lesssim 1 \mathrm{keV}$ were somehow accelerated, the X-ray emission from cooling gas would be reduced. Begelman \& Fabian (1990) and Fabian et al. (2001) propose that hot gas $\left(T_{\text {hot }}\right)$ may rapidly mix with cold gas $\left(T_{\text {cold }}\right)$ and thermalize to $T_{m i x} \sim\left(T_{\text {hot }} T_{\text {cold }}\right)^{1 / 2}$ with little emission from temperatures $T_{\text {hot }}>T>T_{\text {mix }}$. Assuming $T_{\text {hot }} \sim 10^{7} \mathrm{~K}$ and $T_{\text {cold }} \sim 10^{4} \mathrm{~K}$, then $T_{\text {mix }} \sim 3 \times 10^{5}$. One potential difficulty with this process is that the $\sim 10^{4} \mathrm{~K}$ gas in E galaxies has a filling factor of only $\sim 10^{-6}$ (e.g. Mathews 1990) so it is not clear that the hot gas can find enough cold gas to mix with. (Neutral and molecular gas at $T<10^{4}$ $\mathrm{K}$ are also in short supply.) In any case, when the gas at $T_{m i x} \sim 3 \times 10^{5} \mathrm{~K}$ cools 
further, it should radiate strongly in the OVI UV doublet which, at least for NGC 1399, is not observed. Finally, the soft X-ray emission missing from cooling regions may appear somewhere else in the spectrum (Fabian et al. 2002b).

Fujita, Fukumoto \& Okoshi (1997), Fabian et al. (2001) and Morris \& Fabian (2003) describe cooling flows in which the metal (iron) abundance is very inhomogeneous. Regions of enhanced iron abundance, possibly enriched by individual Type Ia supernovae, cool rapidly, reducing the X-ray line emission from intermediate temperatures, as required by XMM. The remaining gas of lower metallicity can also cool with less line emission. This is an attractive idea that could help solve the cooling paradox, but it needs to be tested with detailed flow models. In the presence of strong abundance inhomogeneities, the observed abundance and inferred Type Ia supernova rate will need to be appropriately adjusted. This type of inhomogeneity would differ from successful models of Milky Way enrichment where it is usually assumed that Type Ia enrichment products are widely distributed in the interstellar gas, enriching subsequent stellar generations.

Incomplete mixing of stellar ejecta can also have beneficial results. Mathews \& Brighenti have recently modeled the thermal evolution of dusty gas ejected from red giant (AGB) stars in E galaxies in which the gas is first rapidly heated to the ambient temperature, $\sim 1 \mathrm{keV}$, then cools by thermal electron collisions with the grains. The electron-grain cooling time (Dwek \& Arendt 1992) is faster than normal plasma cooling time, the local galactic dynamical time and the grain sputtering time. Some grains survive in the cooled gas and may account for the central clouds of dusty gas observed in E galaxies. Rapid electron-grain cooling may also be consistent with the weakness of X-ray lines at subvirial temperatures in NGC 4636.

In view of the small mass of gas with $T \lesssim 10^{5} \mathrm{~K}$ in E galaxies, any gas that cools must rapidly convert to dense objects that are difficult to observe, like low mass stars. Star formation is aided by the high pressure and low temperature of the cold gas in E galaxy cores (Ferland, Fabian \& Johnstone 1994; 2002; Mathews \& Brighenti 1999a). In cluster cooling flows there is considerable evidence of ongoing star formation (Allen 1995; McNamara 1997; Cardiel et al. 1998: Crawford et al. 1999; Martel et al. 2002). For the much smaller cooling $\dot{M}$ in E galaxies and their groups the evidence for star formation is less obvious such as the features in optical spectra from a "frosting" of young stars (Mathews \& Brighenti 1999a; Trager et al. 2000).

\section{COOLING FLOWS HEATED BY ACTIVE NUCLEI}

\subsection{Chandra Observations and Entropy Floors}

Instead of devising esoteric constructions to hide the cooling gas, why not simply heat the gas and avoid cooling altogether? This is a good idea, but it is far from proven that it can work. There is certainly enough energy in garden variety active galactic nuclei $(\mathrm{AGN}), \sim \tau 10^{44} \mathrm{ergs} \mathrm{s}^{-1}$, even for duty cycles $\tau$ of a few percent, to balance the total energy radiated from galactic/group cooling flows, $L_{x} \sim 10^{41.5 \pm 1} \mathrm{ergs} \mathrm{s}^{-1}$. The heating idea has received enormous additional support from high resolution Chandra X-ray images that reveal an amazing variety of structural disturbances near the flow centers where, according to current orthodoxy, supermassive black holes can be activated by inflowing gas to become AGNs. So far most Chandra observations are of cluster-centered, E galaxies hav- 
ing strong radio sources, but similar hot gas irregularities are also apparent in the centers of more normal, group-centered E galaxies and seem to be an almost universal phenomenon [M84 (Finoguenov \& Jones 2001), NGC 507 (Forman et al. 2001), NGC 4636 (Jones et al. 2002, Loewenstein \& Mushotzky 2002), NGC 5044 (Buote et al. 2003a).

Of particular interest are the X-ray cavities, regions of much lower thermal emission that are often coincident with radio lobes. In Hydra A/3C295 (McNamara et al. 2000; David et al. 2001; Allen et al 2001) the non-thermal energy density in the radio lobes has apparently displaced the thermal gas. In other cases, the radio-X-ray connection is less clear: Perseus/NGC 1275 (Böhringer et al. 1993; Fabian et al 2000; Churazov et al. 2000), A2052 (Blanton et al. 2001b) or A4059 (Heinz et al. 2002). For example, only one of the two high-contrast central X-ray cavities in Perseus/NGC 1275 is coincident with a maximum in the double-lobed radio emission. The other radio lobe has apparently not displaced the local thermal gas, although relativistic electrons appear to be flowing from this radio lobe into an adjacent X-ray hole. Perseus/NGC 1275 is a particularly interesting case because it contains multitude of X-ray holes located at random azimuthal orientations and distances from the center. Most of the "ghost cavities" at larger distances from the galactic center no longer produce ( $\sim \mathrm{GHz})$ radio emission; either the relativistic electrons have lost their energy or the holes have a different origin. Whether the cavities are dominated by thermal or non-thermal plasma, they must be buoyant on timescales $t_{\text {buoy }} \sim 10^{7}-10^{8}$ yrs (e.g. Churazov et al. 2001). In any case, X-ray cavitation and other irregularities in the central hot gas suggest energy sources capable of heating the cooling flows.

Another manifestation of central heating may be the high entropy typically observed in group scale flows. In a perfect, starless, metal-free, $\Lambda$ CDM, hierarchical universe filled with adiabatic gas and self-similar dark halos, the bolometric Xray bremsstrahlung luminosities of galaxy groups and clusters would scale in a self-similar fashion with gas temperature, $L_{x} \propto T^{2}$ (Kaiser 1986; 1991; Evrard \& Henry 1991). However, in our particular universe this relation is somewhat steeper, $L_{x} \propto T^{3}$ (e.g. Arnaud \& Evrard 1999), and may become very steep (at least $L_{x} \propto T^{4}$ ) for groups having $k T \lesssim 1 \mathrm{keV}$ (Helsdon \& Ponman 2000a,b). Evidently the gas in E-dominant groups has somehow acquired an additional "entropy" $S \equiv T / n_{e}^{2 / 3} \sim 100 \mathrm{keV} \mathrm{cm}{ }^{2}$ in excess of that received in the cosmic accretion shock near the virial radius. This excess has been referred to as the "entropy floor" (Ponman et al. 1999; Lloyd-Davies et al. 2000). More recent studies (Mushotzky et al. 2003; Ponman et al. 2003) claim that the entropy at 0.1 of the virial radius varies as $S_{0.1} \propto T^{0.65}$ with no pause at the $100 \mathrm{kev} \mathrm{\textrm {cm } ^ { 2 }}$ floor for groups and E galaxies; $S_{0.1} \propto T$ would be expected in a perfect adiabatic universe.

The universality of entropy-enhancement in groups has led to the hypothesis that the gas experienced some additional early heating before (or as) it flowed into the group dark halos. Early "pre-heating" by $\sim 1 \mathrm{keV} /$ particle could explain the aberrant behavior of groups in both the $L_{x}-T$ and $S-T$ plots. Although this level of heating would only be apparent in the shallow central potentials of galaxy groups, many authors have adopted a stronger assumption: that all baryonic gas, including gas currently in both groups and clusters, experienced some "non-gravitational" heating (star formation, Pop III stars, AGN, etc.) prior to its entry into the dark halos (e.g. Tozzi \& Norman 2001; Borgani et al 2001, 
2002). Heating at early cosmic times when the density is low is attractive because the entropy can be increased with a smaller energy expenditure per particle. The energy required exceeds that expected from Type II supernovae associated with the formation of the old stellar population in elliptical galaxies and spiral bulges, $\sim 0.2 \mathrm{keV}$ per particle, assuming a Salpeter IMF. Perhaps more attention should be paid to the possibility that much of the low entropy gas was removed by star formation and the remaining gas was heated by star formation (Loewenstein 2000; Brighenti \& Mathews 2001) and by a central AGN after the baryonic gas entered the dark halos.

\subsection{AGN Heating}

The absence of XMM spectral evidence for cooling at the expected level and obvious disturbances in Chandra images are the primary motivations for introducing some type of vigorous heating in the flow models. At the present time, however, both of these motivations are better documented for E galaxies in cluster environments than for similar galaxies in small groups that are more highly constrained. The gasdynamical consequences of heating have been investigated on two spatial scales: (1) attempts to arrest or retard radiative cooling throughout the hot gas and (2) studies of the evolution of small heated regions. We shall discuss these two approaches in turn.

In some models of heated "cooling flows" the physical heating mechanisms shocks, cosmic rays, Compton heating, thermal conductivity etc. - are explicitly invoked, but in others an ad hoc heating is simply imposed on the flow. Thermal conduction has been a popular heating option for many years (e.g. Bertschinger \& Meiksin 1986; Meiksin 1988; Bregman \& David 1988; Rosner \& Tucker 1989). The uncertain influence of magnetic field geometry on the conductivity provides another adjustable parameter to fit the data. If the thermal conduction term in Equation (5) is comparable to the radiative emission term, $n_{e}^{2} \Lambda \sim f 2 \kappa T / r^{2}$, the magnetic suppression factor must be $f \sim 56\left(n_{e} r_{k p c}\right)^{2} T_{7}^{-7 / 2} \sim 0.2 r_{k p c}^{-0.36} T_{7}^{-7 / 2}$ where $n_{e} \approx 0.055 r_{k p c}^{-1.18} \mathrm{~cm}^{-3}$ applies to NGC 4472 for $r_{k p c} \gtrsim 0.3$ (Figure 2a). This level of magnetic suppression is in excellent agreement with $f \sim 0.2$ determined by Narayan \& Medvedev (2001) for thermal conduction in a hot plasma with chaotic magnetic field fluctuations. Conduction could be very important in cluster-centered E galaxies such as M87 that are surrounded by much hotter gas; these galaxies are of interest because they represent galactic scale flows with different outer boundary conditions. Regardless of the heating mechanism, it is essential that the density and temperature profiles in heated flows agree with observations. In particular, the heating must preserve the positive temperature gradients universally observed within several $R_{e}$ (Figure $2 \mathrm{~b}$ ).

\subsection{Cooling Flow Models with Heating}

Nonthermal radiofrequency emission from cosmic ray electrons is observed in the centers of $\sim 50$ percent of giant E galaxies (e.g. Krajnovic \& Jaffe 2002). It is likely that proton cosmic rays are also present or even dominant as in the Milky Way. Cosmic rays could heat the thermal gas either by Coulomb collisions (Rephaeli 1987; Rephaeli \& Silk 1995) or by dissipation of Alfven waves excited as cosmic rays stream along field lines (e.g. Tucker \& Rosner 1983; Rose et al. 1984; Rosner \& Tucker 1989; Begelman \& Zweibel 1994). Loewenstein, Zweibel 
\& Begelman (1991) studied the propagation of cosmic rays in a static hot gas galactic atmosphere having an idealized globally radial magnetic field. They suppose that outwardly streaming cosmic rays heat by wave dissipation at small galactic radii (also see Böhringer \& Morfill 1988) whereas gas at larger radii is heated by thermal conduction. Although their model is successful in producing central regions with $d T / d r>0$, the central gas density gradient $d n / d r$ is flatter than observed because the gas is both supported and heated by cosmic rays. They conclude that this type of cosmic-ray heating cannot balance radiative cooling.

Tabor \& Binney (1993) develop steady state flows in which a convective core heated from the center is attached to an outer cooling inflow. However, in the convective core the temperature gradient is determined by the gravitational potential, $\left(k / \mu m_{p}\right)(d T / d r)=-(2 / 5)(d \Phi / d r)$, and must always be negative, contrary to observation. The gas velocity in the convective core is problematical: if it is negative, all the gas shed from evolving stars, $\sim 10^{10} M_{\odot}$, would cool at the very center; if it is positive or zero, cooling would occur as a galactic drip (Mathews 1997) in compressed gas at the outer boundary of the convective core. Binney \& Tabor (1995) discuss time dependent hot gas flows in E galaxies without dark halos. In addition to central AGN heating, a large number of Type Ia supernovae at early times drive a galactic outflow throughout much of the evolution and help to reduce the mass of cooled gas (as in Ciotti et al. 1991). These supernovae are also likely to enrich the hot gas with iron far in excess of observed abundances. In the supernova heated flows $d T / d r$ is negative throughout the flow evolution, unlike observed profiles, and the most successful models agree with observed gas density profiles only during a brief $\sim 10^{8}$ year period preceding cooling catastrophes when the central gas density increases rapidly due to radiative losses. Binney \& Tabor also consider intermittent AGN heating within $\sim 1 \mathrm{kpc}$ triggered by gas inflow into the center. As SNIa heating subsides and the flow evolves toward a central cooling catastrophe, the AGN heating is activated but the central gas density still continues to increase. As Binney \& Tabor suggest, this may result in distributed cooling and star formation in the central 1-2 kpc. Such radiative cooling might not be possible in a strict interpretation of the XMM spectra.

Tucker \& David (1997) study time dependent heated models of cluster scale flows without cooling dropout or mass supply from stars. Gas near a central AGN is intermittently heated by relativistic electrons when gas flows into the origin. The temperature and entropy gradients become negative and the density gradient is positive. Such flows are both convectively and Rayleigh-Taylor unstable. When Tucker \& David include thermal conduction, the outward heat flux results in stable solutions having cores of nearly flat density that are nearly isothermal. However, recent Chandra and XMM observations of the cluster-centered galaxies M87 in Virgo and NGC 4874 in Coma show that the gas temperature drops precipitously from $3-9 \mathrm{keV}$ to $\sim 1 \mathrm{keV}$ between 50 and $10 \mathrm{kpc}$ of the galactic centers (Böhringer et al. 2001; Molendi \& Gastaldello 2001; Arnaud et al. 2001; Vikhlinin et al. 2001). These steep thermal gradients can be understood with oldfashioned time-dependent cooling flow models with distributed cooling dropout (Brighenti \& Mathews 2002a), but only if thermal conductivity is suppressed $(f \lesssim 0.1)$.

Ciotti \& Ostriker $(1997 ; 2001)$ assume that gas in the cores of isolated elliptical galaxies is Compton heated by powerful isotropic gamma rays $\left(L_{b o l} \sim 10^{46}-10^{48}\right.$ $\left.\operatorname{erg~s}^{-1} ; T_{C} \approx\langle h \nu\rangle / 4 \sim 10^{9} \mathrm{~K}\right)$ whenever mass is accreted into an AGN at the 
center. The explosive expansion of this ultrahot gas drives strong shocks out to $\sim 5 \mathrm{kpc}$ and beyond. The hard continuum is only activated about $\sim 10^{-3}$ of the time, so only one in $\sim 1000$ of known elliptical galaxies should be a gammadominant quasar at any time. For these models Ciotti \& Ostriker also assume a high Type Ia supernova rate and no additional circumgalactic gas. Such hot gas atmospheres - less deeply bound and with small inertial masses - can be significantly modified by AGN heating, reducing the mass of gas that cools near the galactic core. However, $d T / d r$ is generally negative and the gas density has a broad central core, although the density and temperature profiles vary with time. The central core in the X-ray surface brightness distribution varies in size from 1 to $40 \mathrm{kpc}$ which is much larger than the $\sim 0.6 \mathrm{kpc}$ core in NGC 4649, an elliptical having an $L_{B}$ similar to that used in the Compton heated models. It is unlikely, moreover, that gamma ray QSOs exist in all elliptical galaxies or that this radiation is isotropic. AGN sources dominated by gamma rays, discovered with EGRET, are blazars or OVV (optically violent variables) type QSOs having flat radio spectra with superluminal VLBI activity, suggesting that the hard radiation is highly collimated along the line of sight (e.g. von Montigny et al. 1995; Impey 1996; Hartman et al. 1999). Finally, the Compton temperature $T_{C}$ of more typical, more isotropic AGNs and QSOs is less than $10^{7} \mathrm{~K}$, so cooling flow gas is more likely to be Compton cooled than heated (Nulsen \& Fabian 2000).

Kritsuk, et al. (1998; 2001) describe 1D and 2D heated galactic flows having convective cores. The initial state for these flows is a static hot gas atmosphere in which the source terms in equations (3) and (5) dominate, i.e. gas ejected from stars is exactly consumed by distributed radiative dropout $\left(-q \rho / t_{\text {cool }}\right)$ and radiative losses are balanced by stellar and supernova heating (Kritsuk, et al. 1998). When additional central heating is supplied, the core of the flow becomes convective. After $3 \times 10^{8}$ years the temperature gradient becomes negative in the convecting core a few kpc in size, unlike the profiles in Figure 2b. At this time an incipient cooling catastrophe seems to develop where the slowly outflowing convective core confronts the stationary gas beyond, similar to the thermal instabilities in galactic drips (Mathews 1997). Brighenti \& Mathews (2002b) consider a wide variety of $1 \mathrm{D}$ and $2 \mathrm{D}$ cooling flows that are heated within some radius by an unspecified process and triggered by gas flow into the origin. Of the wide variety of heating scenarios considered, none are in reasonable agreement with observed hot gas temperature and density profiles. Even for poor fits to the observations, these models require finely tuned heating scenarios. Idealized flows in which radiative cooling is perfectly balanced by global heating are grossly incompatible with observations. AGN feedback heating often results in spontaneous and spatially distributed cooling produced by non-linear compressions in turbulent regions. Turbulence-induced cooling is associated with quasi-cyclic variations in the hot gas density profile. When cooling flows are partially supported by nonthermal pressure, similar cooling instabilities develop. The global mass cooling rate is not altered by any form of heating considered - in apparent violation of XMM spectra.

Ruszkowski \& Begelman (2002) describe a cluster flow model that is heated by conduction from the outside and the entire flow is instantaneously heated when gas flows into the central AGN. The temperature gradient in these solutions is positive, and this much desired outcome is relatively insensitive to the scale and shape of the region heated by the central AGN. Successful Ruszkowski-Begelman flows require that the thermal conductivity suppression factor be in the range 
$0.1 \lesssim f \lesssim 0.5$. Fortunately this range includes the value $f \approx 0.2$ recently advocated by Narayan \& Medvedev (2001). Flows on galactic scales also benefit from the combined effects of AGN heating and conduction, but the agreement is generally less satisfactory than for cluster flows (Brighenti \& Mathews 2003). Because of the lower temperature in galactic flows, $k T \sim 1 \mathrm{keV}$, marginally acceptable, but not ideal, flow solutions are possible only if the conductivity is close to its full Spitzer value, $f \approx 1$. However, in these solutions the hot gas iron abundance is several times solar throughout most of the flow. This is much higher than observed, even though the current Type Ia supernova rate used is low, $\mathrm{SNu}\left(t_{n}\right)=0.06 \mathrm{SNu}$. Therefore, to fully accept the beneficial combination of heating plus conduction in galactic flows, it is also necessary to hypothesize some means of reducing the computed iron abundance. Some (but not all) of the iron produced by Type Ia may cool before entering the hot gas, some selective cooling removes the excess iron, etc.

In summary, all current attempts to reduce the mass cooling rate $\dot{M}$ in cooling flows by factors or 5 - 10 with various heating mechanisms are inadequate for one reason or another, particularly for flows in E-dominant group gas. When computed for many Gyrs, the heating often has little effect on $\dot{M}$ and the global density and temperature profiles disagree with the observations. Thermal conduction has a helpful role, but is less effective in galactic flows. Essentially static flows cannot be correct either because the hot gas iron enrichment by Type Ia supernovae inside the central E galaxy would be enormous after several Gyrs. Abundances are a major constraint on galactic flows.

\subsection{Models of X-ray Cavities}

Several recent theoretical studies of X-ray cavities have examined the consequences of introducing heated gas in some localized region away from the center of the cooling flow. If the energy density in the cavities is sufficiently large, they will expand supersonically, producing shocks that heat the adjacent cooling flow gas (Clarke, Harris, \& Carilli 1997; Heinz, Reynolds, \& Begelman 1998; Begelman \& Cioffi 1989; Rizza et al. 2000; Reynolds, Heinz, \& Begelman 2001; Soker, White, David \& McNamara 2001). While such violent heating may occur in some situations, recent Chandra and XMM observations indicate that the gas temperature in the rims around the cavities and radio holes is typically cooler, not hotter, than average (Fabian 2001; McNamara 2001). These low-entropy rims cannot be understood as local gas that was shocked and subsequently lost entropy by radiation (Nulsen et al 2002; Soker, Blanton \& Sarazin 2002).

The 2D hot buoyant bubbles computed by Churazov et al. (2001) slowly float upward in the cooling flow atmosphere (also: Saxton et al. 2001; Brüggen \& Kaiser 2001; Brüggen et al. 2002). These rising cavities are accompanied by a column of colder (low entropy) gas that moves radially upward near the center of the bubble. Unless this colder gas is subsequently heated, however, it should eventually fall back. Quilis et al. (2001) studied the evolution of a nearly axisymmetric 3D bubble produced by heated gas at some finite radius, simulating jet heating. They noticed that the buoyant bubble is surrounded by a shell of slightly colder gas which they attributed to cooling expansion as low-entropy gas is pushed by the bubble toward regions of decreasing ambient pressure (Nulsen et al 2002; Soker, Blanton \& Sarazin 2002). Heating by jets can also create buoyant regions with slightly cooler rims that float upwards approximately along the jet 
axis (Reynolds, Heinz, \& Begelman 2001; 2002). Brighenti \& Mathews (2002c) show that central density irregularities and large, randomly oriented X-ray holes may be a natural result of the evolution of a single spherically heated region at the center of a massive E galaxy. The gaseous rims around the holes are cool, as observed, provided the heating occurs in the region of low entropy near the center of the flow and this is illustrated with an analogous similarity solution.

\section{ROTATION AND MAGNETIC FIELDS}

If the classical cooling inflow hypothesis is correct, both rotation and magnetic fields should be amplified near the center of the flow. Mass and angular momentum conservation in the hot gas within slowly rotating elliptical galaxies ultimately results in extended, massive disks of rotationally supported cold gas in the equatorial plane. As the hot interstellar gas approaches the disks, its density and thermal X-ray emission increase, resulting in X-ray images that are considerably flattened toward the equatorial plane out to an effective radius or beyond (Kley \& Mathews 1995; Brighenti \& Mathews 1996; 1997b). Such X-ray disks have never been observed (e.g. Hanlan \& Bregman 2000). This problem can be avoided if the gas is flowing outward, but we have seen that this requires that the heating be fine tuned. Alternatively, cooling inflows that rotate can have nearly circular X-ray images (1) if the mass of hot gas is depleted by spatially extended (multiphase) mass dropout or, in view of XMM observations to the contrary, (2) if angular momentum is transported outward by subsonic turbulent diffusion (Brighenti \& Mathews 2000). If the random velocities of the diffuse $\mathrm{H} \alpha+\mathrm{N}[\mathrm{II}]$ lines, $\sim 150 \mathrm{~km} \mathrm{~s}^{-1}$, are also present in the hot gas, they would be sufficient for this diffusion. Type Ia supernova rates of interest can maintain the centrally observed iron abundance gradient even in the presence of interstellar turbulence sufficient to circularize the X-ray image (Brighenti \& Mathews 2000, Brüggen 2002).

Similarly, seed magnetic fields contained in the ejected envelopes of evolving stars or in relic radio sources can be amplified by the same interstellar turbulence. Ancient fields established by turbulent dynamos during the merging epoch of the E galaxy group could still be present in the hot gas. Further amplification occurs as these fields are compressed by an inward flow of hot gas (Soker \& Sarazin 1990; Moss \& Shukurov 1996; Christodoulou \& Sarazin 1996; Mathews \& Brighenti 1997; Godon, Soker \& White 1998). Field stresses of $\sim 50 \mu \mathrm{G}$, as observed in M87 (e.g. Owen, Eilek \& Keel 1990), formed in this way (without a central AGN) can compete with the thermal pressure in the hot gas and influence the observed gas density gradient. The generic incompatibility of magnetic fields and spherical gravitating atmospheres may give rise to some of the central X-ray surface brightness irregularities observed. Although potentially very important, studies of magnetic fields in cooling flows have been limited by the lack of observations in normal (weak radio) E galaxies and by the theoretical and numerical complexities of field reconnection.

\section{ABUNDANCES IN THE HOT GAS}

Abundance determinations in X-ray astronomy are confused by the ever-changing abundance of the sun. In much of the X-ray literature the "photospheric" iron 
abundance from Anders \& Grevesse (1989) is explicitly or implicitly assumed whereas the currently preferred " meteoritic" value $3.2 \times 10^{-5}$ (by number) is lower by a factor 0.69 (McWilliam 1997; Grevesse \& Sauval 1999). Likewise, the solar abundance of $\mathrm{O}, \mathrm{S}$ and Ar have recently changed by factors of $0.79,1.32$ and 0.69 respectively (Grevesse \& Sauval 1998) and this is offered as a new option in XPEC. To facilitate comparisons between any two X-ray observations, it is essential that authors prominently list the solar abundances assumed, but this has not always been done.

Evidently, at early times the hot gas associated with E galaxies was enriched in metals by Type II supernovae (SNII) that accompanied the formation of the dominant old stellar population. At later times further enrichment has been provided by Type Ia supernovae (SNIa) and material expelled from red giant stars. For a proper cosmic census of supernova enrichment there is no substitute for rich clusters $(\langle k T\rangle \gtrsim 3 \mathrm{keV})$ that are massive enough to retain metal-enriched, SNII-driven galactic winds (e.g. Renzini 1997; 1999). In group-centered elliptical galaxies most of the iron produced by SNII has been dispersed by a galactic wind into distant group gas or nearby intergalactic regions where the gas density is so low that it cannot be observed even with XMM (Brighenti \& Mathews 1999a). Groups are not closed boxes. Chandra observations by Martin, Kobulnicky \& Heckman (2002) of the dwarf starburst wind in NGC 1569 verify that these winds transport almost all of the SNII enrichment into the local intergalactic medium (Vader 1986). Early epoch winds from both dwarf and giant galaxies were undoubtedly even stronger than those in local starburst galaxies. Rampressure stripping may also be relevant to hot gas enrichment (e.g. Toniazzo \& Schindler 2001). Finally, ongoing metal-enriched galactic outflows energized by SNIa are energetically likely in low luminosity ellipticals, $L_{B} \lesssim L_{B \text {,crit }}$.

Yields from Type II supernovae $y_{I I}$ (expressed in $M_{\odot}$ ) vary with initial main sequence stellar mass (e.g. Hamuy 2003) and must be averaged over the initial mass function. While yields for elements that can be identified in X-ray spectra are still quite uncertain (e.g. Gibson, Loewenstein \& Mushotzky 1997; Dupke \& White 2000; Gastaldello \& Molendi 2002), there are three broad categories: the ratio $y_{I a} /\left\langle y_{I I}\right\rangle$ is about 10 for iron, $\sim 1$ for silicon and sulfur (as well as argon, calcium and nickel), and $\sim 0.1-0.2$ for oxygen and magnesium. Note that $y_{I a} /\left\langle y_{I I}\right\rangle$ is far from uniform among the $\alpha$ elements $\mathrm{O}, \mathrm{Mg}, \mathrm{Ar}, \mathrm{Ca}, \mathrm{S}$, and Si. Ideally, it should be possible to account for any observed abundance with a linear combination of SNII and SNIa yields, but current observations may be too uncertain to permit this. Abundances differ according to the quality of the observation, the relative isolation of the $\mathrm{K} \alpha$ or other prominent lines for a given element, the assumptions made about the distribution of gas temperature at every radius in the flow (1T, $2 \mathrm{~T}$, etc.), and possibly also the plasma code (Gastaldello \& Molendi 2002).

In principle, abundances in the hot gas within the stellar images of E galaxies can provide critically important information about the direction and magnitude of the systematic radial flow of hot gas, $\rho u 4 \pi r^{2}$, which is also sensitive to distributed cooling. The known stellar density $\rho_{*}(r)$ and metallicity $z_{*}(r)$ define the source function for metal ejection. However, to extract this flow information one must combine uncertain observations with uncertain supernova yields and with uncertainties in the fraction of metal-enriched gas from SNIa and stellar winds that ultimately goes into the hot phase.

Nevertheless, most X-ray observers currently agree that the hot gas within 
and near group and cluster centered E galaxies has been enriched by SNIa (e.g. Matsumoto et al. 1996; Fukazawa et al. 1998; Dupke \& White 2000; Finoguenov \& Jones 2000; Buote 2000a, 2002; De Grandi \& Molendi 2001; Buote et al. 2003b; Lewis, Stocke \& Boute 2002). The best observed E galaxy is M87 centered in the Virgo cluster (Matsumoto et al. 1996: Finoguenov et al. 2002; Gastaldello \& Molendi 2002), but the presence of a central AGN and X-ray jet in M87 may have disturbed the normal evolution of the hot gas. Similar XMM observations of somewhat lower accuracy are available within a few $100 \mathrm{kpc}$ of the central E galaxies in Abell 496 ( $T \sim 2-5 \mathrm{keV}$; Tamura et al. 2001), NGC 1399 (Buote 2002) and NGC 5044 ( $T \sim 0.7-1.2 \mathrm{keV}$; Buote et al. 2003b). No XMM abundances for NGC 4472 or other normal, group-centered E galaxies have been published as of the Fall of 2002. In all galaxies observed so far the hot gas iron abundance increases toward the central elliptical, rising from $\sim 0.2-0.4$ solar at $\sim 100 \mathrm{kpc}$ to $\sim 1$ solar or more at the center, similar to the stellar abundances there. [Older very subsolar central iron abundances determined with ASCA are now known to be wrong because the radial temperature variation was neglected (the "Fe bias": Trinchieri et al. 1994; Buote \& Fabian 1998; Buote 1999).] The scale of the iron enhanced region, $\sim 50-100 \mathrm{kpc}$, is larger than the half-light radius $R_{e}$ of the central E (cD) galaxy.

Because oxygen and magnesium are formed in the outer layers of massive stars during normal stellar evolution, their Type II supernova yields should be reliable apart from uncertainties in the IMF and metallicity. Consequently, one would expect $\mathrm{O}$ and $\mathrm{Mg}$ abundances to vary together. Although XMM abundances of oxygen and magnesium are less accurate in the hot gas, they typically increase very little toward the core of the central elliptical and often become flat or even decline near the very center (Tamura et al. 2001; Buote et al. 2003b; Johnstone et al. 2002; Gastaldello \& Molendi 2002). NGC 1399 and NGC 5044 data show that $\mathrm{Mg}$ traces $\mathrm{Si}$ and $\mathrm{S}$ more closely than $\mathrm{O}$; only $\mathrm{O}$ seems flat. Either the $\mathrm{O}$ abundance in the mass-losing stars is surprisingly similar to that in the ambient hot gas, or the $\mathrm{O}$ expelled from O-rich stars is not all going into the hot gas.

The hot gas abundance $z_{i}$ (by mass in solar units) of element $i$ should vary as

$$
\frac{d z_{i}}{d t} \equiv \frac{\partial z_{i}}{\partial t}+u \frac{\partial z_{i}}{\partial r}=\left(z_{*, i}-z_{i}\right) \alpha_{*} \frac{\rho_{*}}{\rho}+\frac{1.4 y_{I a, i}}{z_{i, \odot} M_{s n}} \alpha_{s n} \frac{\rho_{*}}{\rho}
$$

where $y_{I a, i} / M_{s n}$ is the fraction of mass in element $i$ ejected by Type Ia supernovae, $z_{i, \odot}$ is its solar abundance and 1.4 is the ratio of total to hydrogen mass at solar abundance. This equation is quite general, but it is interesting to apply it to subsonic galaxy/group inflows or outflows in which the gas density $\rho(r)$ remains essentially constant for several Gyrs. We assume that all stellar and supernova ejecta goes into the hot gas. In a Lagrangian frame moving slowly with the gas velocity $u$, the abundance of element $i$ in the gas increases from some initial value $z_{0, i}$ to an asymptotic limit

$$
z_{e q, i}(r)=z_{*, i}(r)+\frac{1.4 y_{I a, i}}{z_{i, \odot} M_{s n}} \frac{\alpha_{s n}}{\alpha_{*}} .
$$

This equilibrium abundance is reached after $t_{e q}=\left(\rho / \rho_{*}\right) / \alpha_{*}=2.80 \times 10^{8} r_{k p c}^{1.18}$ yrs, using NGC 4472 densities from Figure 2a.

For iron enrichment $\left(y_{I a, F e}=0.7 M_{\odot} ; M_{s n}=1.4 M_{\odot} ; \mathrm{SNu}\left(t_{n}\right)=0.06 \mathrm{SNu}\right.$; $z_{\odot, F e}=1.83 \times 10^{-3}$ by mass) we find $z_{e q, F e}=z_{*, F e}+2.35$ solar. This value is 
similar to the gas abundance in the isolated solution shown in the first column of Figure 5 in which $z_{F e}$ decreases from $\sim 3$ solar at the center to $\sim 2$ at large radii where $z_{*, F e}$ becomes small. It is significant that this flow solution overestimates the iron abundance observed in NGC 4472, particularly for $r \gtrsim 10 \mathrm{kpc}$. The correct iron abundance gradient in $10 \lesssim r \lesssim 50 \mathrm{kpc}$ is, however, nicely fit by the solution that includes circumgalactic gas (dashed line, first column, Fig. 5). This agreement is only possible if the low metallicity circumstellar (or cluster) gas is flowing inward, diluting the entire flow, i.e. $z_{F e}$ increases toward $z_{e q, F e}$ as the gas flows in. Of course inflow is only possible if some (distributed) cooling occurs. If the global radial flow is outward, the circumgalactic gas (with low $z_{F e}$ ) would be pushed outward and the Fe abundance would approach $\sim z_{e q, F e}$ within $r \approx 3 t_{G y r}^{0.85}$ $\mathrm{kpc}$. In this case the hot gas iron abundance $z_{F e}(r)$ would be at least as large as that of the isolated flow solution (column 1, Fig. 5) which clearly exceeds the iron abundance observed in NGC 4472. The observed iron abundance gradient is therefore a powerful argument for systematically inflowing hot gas, similar to the inflow required to explain the gas temperature profile $(d T / d r>0)$.

The abundance of oxygen in the hot gas, which comes mainly from stellar mass loss $\left(y_{I a, O} \approx 0\right)$, should approach the stellar abundance, $z_{e q, O}=z_{*, O}$ in time $t_{e q}$. The $\mathrm{O}$ abundance in M87 rises gradually with $\rho_{*}$, which is consistent with some stellar enrichment, but it is curious that the $\mathrm{O} / \mathrm{Fe}$ ratio is almost constant with galactic radius (Gastaldello \& Molendi 2002). The small $\mathrm{O}$ abundance $z_{O} \sim 0.3$ observed in NGC 5044 and M87 (Buote et al. 2003b; Gastaldello \& Molendi 2002) suggests that only a fraction of the gas lost from stars may go into the hot phase. Alternatively, the stellar $\mathrm{O}$ abundance in E galaxies may be subsolar, as suggested by observations of forbidden emission lines in E galaxy planetary nebulae (Jacoby \& Ciardullo 1999; Walsh et al. 1999), although nebular forbidden lines are known to give lower $\mathrm{O}$ abunances than the recombination lines. But $\mathrm{O}$ and $\mathrm{Mg}$ are expected to vary in a similar manner and stellar $\mathrm{Mg}$ is known to increase with $\rho_{*}$ (Trager et al. 2000). If the $\mathrm{O}$ abundance is systematically low in $\mathrm{E}$ galaxies, this may indicate the presence of hypernovae in which oxygen is burned into heavier elements (Loewenstein 2001).

One of the most remarkable peculiarities in the abundance profiles are the central minima observed for almost all elements in M87 (Gastaldello \& Molendi 2002), in NGC 4636 (Loewenstein \& Mushotzky), in AWM 7 (Johnstone et al. 2002) and elsewhere. For ellipticals that are resolved by ROSAT within $5 \mathrm{kpc}$, the observations compiled by Buote $(2000 \mathrm{a}, \mathrm{c})$ indicate that the iron abundance is often flat (NGC 1399) or decreasing (NGC 4472; NGC 5846; NGC 4636) within $5 \mathrm{kpc}$, assuming no intrinsic absorption. Only NGC 4649 shows an increase. Subsolar central hot gas iron abundances, $\sim 0.8$ solar, for $r \lesssim 3 \mathrm{kpc}$ have been confirmed with XMM RGS observations (Xu et al. 2002; Sakelliou et al 2002). It has been realized for some time that the central abundances could appear lower if the prominent X-ray resonance lines emitted there were optically thick and diffused outward before escaping (Gil'fanov et al. 1987, Tawara et al. 1997, Shigeyama 1998; Böhringer et al. 2001; Mathews et al. 2001; Sazonov et al. 2002). However, the XMM RGS spectra of Abell 496 (Tamura et al. 2001) and M87 (Gastaldello \& Molendi 2002) show central reversals even for non-resonant lines. In M87 the optical depth may be too low for line radiative transfer to be important, particularly if the hot gas is mildly turbulent (Mathews et al. 2001; Sakelliou et al. 2002). Morris \& Fabian (2003) describe a transient enrichment process that produces a central minimum but this feature would probably disap- 
pear if the source function were constant with time. There is at present no fully satisfactory explanation of these strange abundance profiles, assuming they are real.

We thank David Buote for reading an early draft of this review and for his helpful suggestions. Studies of the evolution of hot gas in elliptical galaxies at UC Santa Cruz are supported by NASA grants NAG 5-8409 \& NAG 5-9956 and NSF grants AST-9802994 \& AST-0098351 for which we are very grateful. FB is supported in part by grant MURST-Cofin 00.

\section{List of Figures}

A plot of the bolometric X-ray luminosity and B-band optical luminosity for elliptical galaxies ( $\mathrm{RC} 3$ type $\mathrm{T} \leq-4)$ from the compilation of O'Sullivan et al. (2001). X-ray detections are shown with filled circles and upper limits with open triangles. The dashed line is an approximate locus of the total luminosity $L_{x, *} \propto L_{B}$ of stellar and other discrete sources also from O'Sullivan et al. . . . . . . . .

(2a Left panel:) The observed and azimuthally averaged electron density profile $n(r)$ in NGC 4472 is shown as a function of radius normalized to the effective radius $R_{e}=8.57 \mathrm{kpc}$ at distance $d=17$ Mpc. The observations are from Einstein (Trinchieri, Fabbiano, \& Canizares 1986) (filled circles) and ROSAT (Irwin \& Sarazin 1996) (open circles); for the inner region we have Abel-inverted Chandra surface brightness data from Loewenstein et al. (2001) (open squares) and normalized them to previous observations. The solid line is an analytic fit to the observations. The dashed line is the square root of the stellar density $\rho_{*}^{1 / 2}(r)$ normalized to $n$ at $r=R_{e}$. (2b Right panel:) Typical temperature profiles for several bright E galaxies, taken from Brighenti \& Mathews (1997a), based on the following sources: NGC 1399: ROSAT PSPC from Jones et al. (1997); NGC 5044: ROSAT PSPC from David et al. (1994); NGC 4636: ROSAT PSPC from Trinchieri et al. (1994); NGC 4472: ROSAT HRI AND PSPC from Irwin \& Sarazin (1996). The solid line is an approximate analytic fit to $T(r)$ for NGC 4472. . . 11

(3a Left panel:) The total mass $M_{\text {tot }}(r)$ for NGC 4472 (solid line) is found from Equation (1) with $P_{n t}=0$ using the solid line approximations to the X-ray observations in Figure 2. The total mass of hot gas $M_{\text {gas }}(r)$ (dot-dashed line) is relatively small. The stellar mass profile $M_{*}(r)$ (long dashed line) is based on a de Vaucouleurs plus core profile with mass to light ratio $\Upsilon_{B}=7$. The NFW dark halo profile $M_{n f w}(r)$ (short dashed line) corresponds to a total mass of $4 \times 10^{13} M_{\odot}$. (3b Right panel:) Line of sight stellar velocity dispersion profiles $\sigma(\beta ; R)$ as a function of projected radius $R$. The curves are computed from solutions $\sigma_{r}(r)$ of Equation (2) assuming constant $\beta$ which labels each curve. The observations are from Fried \& Illingworth (1994). . . . . . . . . . . . . . . . 12

Contours show a combined ROSAT HRI and PSPC X-ray image of NGC 4472 superimposed on an optical image from the Digital Sky Survey (Irwin \& Sarazin 1996). . . . . . . . . . . . . 13 
A variety of computed time dependent galactic flow models are compared at time $t_{n}=13$ Gyrs with the observed gas density (top row) and temperature (middle row) of NGC 4472 taken from Figure 2. The bottom row shows the computed hot gas iron abundance compared with the observations of Buote (2000c). First column: A plot of the profiles at $t_{n}$ for an approximate model of NGC 4472 assuming an isolated galaxy (solid lines); this "reference" flow is based on an assumed parameter set as explained in the text. A similar galactic flow but including initial circumgalactic gas ("cgg") is also shown (dashed lines). Second column: A model similar to the reference flow but with ad hoc spatially distributed cooling with dropout parameter $q=1$. Third column: Two flows similar to the reference flow (for which $\mathrm{SNu}\left(t_{n}\right)=0.06$ ) but with additional heating by Type Ia supernovae: $\mathrm{SNu}\left(t_{n}\right)=0.18$ (solid lines) and $\mathrm{SNu}\left(t_{n}\right)=0.25$ (dashed lines). Fourth column: Three galactic flows similar to the reference flow but calculated with different past Type Ia supernova rates $\mathrm{SNu}(t) \propto t^{-s}: s=0$ (solid lines), 1.5 (dashed lines) and 2 (dotted lines). In the reference model $s=1.3$.

Literature Cited

Abramowicz MA, Chen X, Kato S, Lasota J-P, Regev O. 1995. Ap. J. 438:L37-39

. Allen SW. 1995. MNRAS 276:947-60

. Allen SW. 2000. MNRAS 315:269-95

- Allen SW, Fabian AC, Edge AC, Bautz MW, Furuzawa A, Tawara Y. 1996. MNRAS 283:263-81

- Allen SW, Fabian AC. 1997. MNRAS 286:583-603

. Allen SW, Taylor GB, Nulsen PEJ, Johnstone RM, David LP, et al. 2001. MNRAS 324:842-58

- Anders E, Grevesse N. 1989. Geochim. Cosmochim. Acta 53:197-214

- Arnaud M, Aghanim N, Gastaud R, Neumann DM, Lumb D, et al. 2001. Astron. Astrophys. 365:L67-73

. Arnaud M, Evrard AE. 1999. MNRAS 305:631-40

- Athey AE, Bregman JN, Bregman JE, Temi P, Sauvage M. 2002. Ap. J. 571:272-81

Bailey ME. 1980. MNRAS 191:195-206

. Balbus SA. 1991. Ap. J. 372:25-30

. Begelman MC, Cioffi DF. 1989 Ap. J. 345:L21-24

. Begelman MC, Fabian AC. 1990. MNRAS 244:26P-29P

. Begelman MC, Zweibel EG. 1994. Ap. J. 431:689-704

. Bender R. 1996. In New Light on Galaxy Evolution. IAU Symp 171, ed. R. Bender and RL. Davies (Kluwer Academic Publishers). 181-90

. Bertin G, Toniazzo T. 1995. Ap. J. 451:111-24

. Bertschinger E, Meiksin A. 1986. Ap. J. 306:L1-5

. Beuing J, Dobereiner S, Bohringer H, Bender R. 1999. MNRAS 302:209-21

. Binette L, Magris CG, Stasinska G, Bruzual AG. 1994. Astron. Astrophys. 292:13-19

. Binney JJ, Mamon GA. 1982. MNRAS 200:361-75

. Binney JJ, Tabor G. 1995. MNRAS 276:663-78

. Blandford RD, Begelman MC. 1999. MNRAS 303:L1-5

. Blanton EL, Sarazin CL, Irwin JA. 2001. Ap. J. 552:106-19

. Blanton EL, Sarazin CL, McNamara BR, Wise MW. 2001. Ap. J. 558:L15-18

. Böhringer H, Belsole E, Kennea J, Matsushita K, Molendi S, et al. 2001. Astron. Astrophys. 365:L181-87

. Böhringer H, Matsushita K, Churazov E, Ikebe Y, Chen Y. 2002. Astron. Astrophys. 382:804-20

. Böhringer H, Morfill GE. 1988. Ap. J. 330:609-19

. Böhringer H, Voges W, Fabian AC, Edge AC, Neumann DM. 1993. MNRAS 264:L25-28

. Borgani S, Governato F, Wadsley J, Menci N, Tozzi P, et al. 2001. Ap. J. 559:L71-74

. Borgani S, Governato F, Wadsley J, Menci N, Tozzi P, et al. 2002. MNRAS 336:409-24 
. Bregman JN, David LP. 1988. Ap. J. 326:639-44

. Bregman JN, McNamara BR, O'Connell RW. 1990. Ap. J. 351:406-11

. Bregman JN, Miller ED, Irwin JA. 2001. Ap. J. 553:L125-28

. Bregman JN, Snider BA, Grego L, Cox CV. 1998. Ap. J. 499:670-76

. Brighenti F, Mathews WG. 1996. Ap. J. 470:747-61

. Brighenti F, Mathews WG. 1997a. Ap. J. 486:L83-86

. Brighenti F, Mathews WG. 1997b. Ap. J. 490:592-604

. Brighenti F, Mathews WG. 1998. Ap. J. 495:239-50

. Brighenti F, Mathews WG. 1999a. Ap. J. 512:65-78

. Brighenti F, Mathews WG. 1999b. Ap. J. 515:542-57

. Brighenti F, Mathews WG. 2000. Ap. J. 539:675-86

. Brighenti F, Mathews WG. 2001. Ap. J. 553:103-20

. Brighenti F, Mathews WG. 2002a. Ap. J. 567:130-43

. Brighenti F, Mathews WG. 2002b. Ap. J. 573:542-61

. Brighenti F, Mathews WG. 2002c. Ap. J. 574:L11-15

. Brighenti F, Mathews WG. 2003. Ap. J. (in press)

. Brown BA, Bregman JN. 1998. Ap. J. 495:L75-78

. Brown BA, Bregman JN. 2000. Ap. J. 539:592-602

. Brown BA, Bregman JN. 2001. Ap. J. 547:154-61

. Brown TM, Ferguson HC, O'Connel RW, Ohl RG. 2002. Ap. J. 568:L19-22

. Brüggen M. 2002. Ap. J. 571:L13-16

. Brüggen M, Kaiser CR. 2001. MNRAS 325:676-84

. Brüggen M, Kaiser CR, Churazov E, Ensslin TA. 2002. MNRAS 331:545-55

. Bullock JS, Kolatt TS, Sigad Y, Somerville RS, Kravtsov AV, Klypin AA, Primack JR, Dekel A. 2000. MNRAS 321:559-75

. Buote DA. 1999. MNRAS 309:685-714

. Buote DA. 2000a. MNRAS 311:176-200

. Buote DA. 2000b. Ap. J. 532:L113-16

. Buote DA. 2000c. Ap. J. 539:172-86

. Buote DA. 2000d. Ap. J. 544:242-57

. Buote DA. 2001. Ap. J. 548:652-70

. Buote DA. 2002. Ap. J. 574:L135-38

. Buote DA, Canizares CR. 1996. Ap. J. 468:184-90

. Buote DA, Canizares CR. 1997. Ap. J. 474:650-58

. Buote DA, Canizares CR. 1998. MNRAS 298:811-23

. Buote DA, Fabian AC. 1998. MNRAS 296:977-94

. Buote DA, Jeltema TE, Canizares CR, Garmire GP. 2002a. Ap. J. 577:183-96

. Buote DA, Lewis AD, Brighenti F, Mathews WG. 2003a. Ap. J. 594:741-57

- Buote DA, Lewis AD, Brighenti F, Mathews WG. 2003b. Ap. J. , in press (astro-ph/0303054)

. Buson LM, Sadler EM, Zeilinger WW, Bertin G, Bertola F, et al. 1993. Astron. Astrophys. 280:409-425

. Canizares CR, Fabbiano G, Trinchieri G. 1987. Ap. J. 312:503-13

. Caon N, Macchetto D, Pastoriza M. 2000. Ap. J. Suppl. 127:39-58

. Caon N, Pastoriza M, Macchetto D. 2001. Astroph. Space Sci. Suppl. 277:409-412

. Cappellaro E, Evans R., Turatto M. 1999. Astron. Astrophys. 350:459-66

- Cardiel N, Gorgas J, Aragon-Salamanca A. 1998. MNRAS 298:977-96

. Carollo CM, de Zeeuw PT, van der Marel RP, Danziger IJ, Qian EE. 1995. Ap. J. 441:L25-28

. Churazov E, Brüggen M, Kaiser CR, Böhringer H, Forman W. 2001. Ap. J. 554:261-73

. Churazov E, Forman W, Jones C, Böhringer H. 2000. Astron. Astrophys. 356:788-94

. Ciotti L, D'Ercole A, Pellegrini S, Renzini A. 1991. Ap. J. 376:380-403

. Ciotti L, Ostriker JP. 1997. Ap. J. 487:L105-08

. Ciotti L, Ostriker JP. 2001. Ap. J. 551:131-52

. Clarke DA, Harris DE, Carilli CL. 1997. MNRAS 284:981-93

. Colbert JW, Mulchaey JS, Zabludoff AI. 2001. Astron. J. 121:808-19

. Crawford CS, Allen SW, Ebeling H, Edge AC, Fabian AC. 1999. MNRAS 306:857-96

. Cristodoulou DM, Sarazin CL. 1996 Ap. J. 463:80-94

. David LP, Jones C, Forman W, Daines S. 1994. Ap. J. 428:544-54

. David LP, Nulsen PEJ, McNamara BR, Forman W, Jones C, et al. 2001. Ap. J. 557:546-59

. Davis DS, White RE III. 1996. Ap. J. 470:L35-39 
. De Grandi S, Molendi S. 2001. Ap. J. 551:153-59

. de Jong T, Norgaard-Nielsen HU, Jorgensen HE, Hansen L. 1990. Astron. Astrophys. 232:317-22

. D'Ercole A, Ciotti L. 1998. Ap. J. 494:535-45

. Di Matteo T, Quataert E, Allen SW, Narayan R, Fabian AC. 2000. MNRAS 311:507-21

. Di Matteo T, Allen SW, Fabian AC, Wilson AS, Young AJ. 2003. Ap. J. 582:133-40 (astro$\mathrm{ph} / 0202238)$

. Di Matteo T, Johnstone RM, Allen SW, Fabian AC. 2001. Ap. J. 550:L19-23

. Djorgovski S, Davis M. 1987. Ap. J. 313:59-68

. Donahue M, Voit GM. 1991. Ap. J. 381:361-72

. Donahue M, Voit GM. 1997. In Galactic and Cluster Cooling Flows, ASP Conf. Ser., ed. N. Soker, 115:48-58. San Francisco: Publ. Astron. Soc. Pac.

. Donnelly RH, Faber SM, O'Connell RM. 1990. Ap. J. 354:52-57

. Dressler A, Lynden-Bell D, Burnstein D, Davies RL, Faber SM, et al. 1987. Ap. J. 313:42-58

. Dupke RA, White RE III. 2000. Ap. J. 528:139-44

. Dwek E, Arendt RG. 1992. Annu. Rev. Astron. Astrophys. 30:11-50

. Eke VR, Navarro JF, Frenk CS. 1998. Ap. J. 503:569-92

. Ekers RD, Kotanyi CG. 1978. Ap. J. 67:47-50

. Emsellem E, Dejonghe H, Bacon R. 1999. MNRAS 303:495-514

. Eskridge PB, Fabbiano G, Kim D-W. 1995a. Ap. J. Suppl. 97:141-84

. Eskridge PB, Fabbiano G, Kim D-W. 1995b. Ap. J. 442:523-37

. Eskridge PB, Fabbiano G, Kim D-W. 1995c. Ap. J. 448:70-83

. Evrard AE, Henry JP. 1991. Ap. J. 383:95-103

. Fabbiano G. 1989. Annu. Rev. Astron. Astrophys. 27:87-138

. Fabbiano G, Kim D-W, Trinchieri G. 1992. Ap. J. Suppl. 80:531-644

. Faber SM, Tremaine S, Ajhar EA, Byun Y-I, Dressler A, et al. 1997. Astron. J. 114:1771-96

. Fabian AC. 1994. Annu. Rev. Astron. Astrophys. 32:277-318

. Fabian AC. 2001. talk at Oxford Radio Galaxies Conference, Aug. 2000 (astro-ph/0103392)

. Fabian AC, Allen SW, Crawford CS, Johnstone RM, et al. 2002b. MNRAS 332:L50-54

. Fabian AC, Canizares CR. 1988. Nature 333:829-31

. Fabian AC, Canizares CR, Böhringer H. 1994. Ap. J. 425:40-42

. Fabian AC, Celotti A, Blundell KM, Kassim NE, Perley RA. 2002a. MNRAS 331:369-75

. Fabian AC, Mushotzky RF, Nulsen PEJ, Peterson JR. 2001. MNRAS 321:L20-24

. Fabian AC, Nulsen PEJ, Canizares CR. 1984. Nature 310:733-40

. Fabian AC, Rees MJ. 1995 MNRAS 277:L55-58

. Fabian AC, Sanders JS, Ettori S, Taylor GB, Allen SW, et al. 2000. MNRAS 318:L65-68

. Fabian AC, Stewart GC, Nulsen PEJ, Itoh H, Canizares CR. 1984. Nature 307:343-46

. Ferland GJ, Fabian AC, Johnstone RM. 1994. MNRAS 266:399-411

. Ferland GJ, Fabian AC, Johnstone RM. 2002. MNRAS 333:876-84

. Ferrari F, Pastoriza MG, Macchetto FD, Bonatto C, Panagia N, Sparks WB. 2002. Astron. Astrophys. 389:355-66

. Ferrari F, Pastoriza MG, Macchetto FD, Caon N. 1999. Astron. Astrophys. Suppl. 136:269-84

. Finoguenov A, Jones C. 2000. Ap. J. 539:603-17

. Finoguenov A, Jones C. 2001. Ap. J. 547:L107-10

. Finoguenov A, Matsushita K, Bohringer H, Ikebe Y, Arnaud M. 2002. Astron. Astrophys. 381:21-31

. Forman W, Markevitch M, Jones C, Vikhlinin A, Churazov E. 2001. in Galaxy Clusters and the High Redshift Universe Observed in X-rays, Proc. of XXI Moriond Conference ed. D Neumann, F. Durret \& J. Tran Thanh Van. (in press) (astro-ph/0110087)

. Fried JW, Illingworth GD. 1994. Astron. J. 107:992-1002

. Fukazawa Y, Makishima K, Tamura T, Ezawa H, Xu H, et al. 1998. Publ. Astron. Soc. Japan 50:187-93

. Fukugita M, Peebles PJE. 1999. Ap. J. 524:L31-33

. Fujita Y, Fukumoto J, Okoshi K. 1997. Ap. J. 488:585-94

. Gal-Yam A, Maoz D, Sharon K. 2002. MNRAS 332:37-48

. Garrington ST, Conway RG. 1991. MNRAS 250:198-208

. Garrington ST, Leahy JP, Conway RG, Laing RA. 1988. Nature 331:147-49

. Gastaldello F, Molendi S. 2002. Ap. J. 572:160-68

. Gebhardt K, Richstone D, Ajhar EA, Lauer TR, Byun Y-I, et al. 1996. Ap. J. 112:105-13

. Georgakakis A, Hopkins AM, Caulton A, Wiklind T, Terlevich AI, Forbes DA. 2001. MNRAS 
326:1431-40

. Gerhard O, Jeske G., Saglia RP. 1998. MNRAS 295:197-215

. Gerhard O, Kronawitter A, Saglia RP, Bender R. 2001. Ap. J. 121:1936-51

. Gibson BK, Loewenstein M, Mushotzky RF. 1997. MNRAS 290:623-28

. Gil'fanov MR, Sunyaev RA, Churazov EM. 1987 Sov. Astron. Lett.13:3-7

. Godon P, Soker N, White RE III. 1998. Astron. J. 116:37-43

. Goudfrooij P, de Jong T. 1995. Astron. Astrophys. 298:784-98

. Goudfrooij P, Hansen L, Jorgensen HE, Norgaard-Nielsen, HU. 1994. Astron. Astrophys. Suppl.105:341-83

. Grevesse N, Sauval AJ. 1998. Space Sci. Rev. 85:161-74

. Grevesse N, Sauval AJ. 1999. Astron. Astrophys. 347:348-54

. Hamuy M. 2003. Ap. J. 582:905-14 (astro-ph/0209174)

. Hanlan PC, Bregman JN. 2000. Ap. J. 530:213-21

. Hartman RC, Bertsch DL, Bloom SD, Chen AW, Deines-Jones P, et al. 1999. Ap. J. Suppl. 123:79-202

. Hattori M, Habe A. 1990. MNRAS 242:399-418

. Hattori M, Yoshida, Habe A. 1995. MNRAS 275:1195-210

. Heckman TM, Baum SA, van Breugel WJM, McCarthy P. 1989. Ap. J. 338:48-77

. Heinz S, Choi Y-Y, Reynolds CS, Begelman M. 2002. Ap. J. 569:L79-82

. Heinz S, Reynolds CS, Begelman M. 1998. Ap. J. 501:126-36

. Helsdon SF, Ponman TJ. 2000a. MNRAS 315:356-70

. Helsdon SF, Ponman TJ. 2000b. MNRAS 319:933-38

. Helsdon SF, Ponman TJ, O'Sullivan E, Forbes DA. 2001. MNRAS 325:693-706

. Henriksen M, Cousineau S. 1999. Ap. J. 511:595-611

. Hernquist L, Barnes JE. 1991. Nature 354:210-12

. Hillebrandt W, Niemeyer JC. 2000. Annu. Rev. Astron. Astrophys. 38:191-230

. Huchtmeier WK. 1994. Astron. Astrophys. 286:389-94

. Huchtmeier WK, Sage LJ, Henkel C. 1995. Astron. Astrophys. 300:675-86

. Impey CD. 1996. Astron. J. 112:2667-2683

. Irwin JA, Sarazin CL. 1996. Ap. J. 471:683-93

. Irwin JA, Sarazin CL. 1997. Astron. J. 113:1580-4

. Irwin JA, Sarazin CL. 1998. Ap. J. 499:650-69

. Jacoby GH, Ciardullo R. 1999. Ap. J. 515:169-90

. Johnstone RM, Allen SW, Fabian AC, Sanders Js. 2002. MNRAS 336:299-308

. Jones C, Forman W, Vikhlinin M, David A, Warmflash A, et al. 2002. Ap. J. 567:L115-18

. Jones C, Stern C, Forman W, Breen J, David L, Tucker W, Franx M. 1997. Ap. J. 482:143-55

. Kaastra JS, Ferrigno C, Tamura T, Paerels FBS, Peterson JR, Mittaz JPD. 2001. Astron. Astrophys. 365:L99-103

. Kaiser N. 1986. MNRAS 222:323-45

. Kaiser N. 1991. Ap. J. 383:104-11

. Keeton CR. 2001. Ap. J. 561:46-60

. Kleijn GAV, Baum SA, de Zeeuw PT, O'Dea CP. 2002. Astron. J. 123:1334-356

. Kley W, Mathews WG. 1995. Ap. J. 438:100-07

Knapp GR, Turner EL, Cunniffe PE. 1985. Astron. J. 90:454-68

. Knapp GR, Gunn JE, Wynn-Williams CG. 1992. Ap. J. 399:76-93

. Knapp GR, Rupen MP. 1996. Ap. J. 460:271-83

. Kobayashi C, Tsujimoto T, Nomoto K. 2000. Ap. J. 539:26-38

. Kormendy J, Bender R. 1996. Ap. J. 464:L119-22

. Krajnovic D, Jaffe W. 2002. Astron. Astrophys. 390:423-30

. Kritsuk AG. 1992. Astron. Astrophys. 261:78-84

. Kritsuk AG, Böhringer H, Müller E. 1998. MNRAS 301:343-56

. Kritsuk AG, Plewa T, Müller E. 2001. MNRAS 326:11-22

- Kronawitter A, Saglia RP, Gerhard O, Bender R. 2000. Astron. Astrophys. Suppl.144:53-84

- Lauer TR, Faber SM, Ajhar EA, Grillmair CJ, Scowen PA. 1998. Astron. J. 116:2263-86

. Lewis AD, Stocke JT, Buote DA. 2002. Ap. J. 573:L13-17

. Lloyd-Davies EJ, Ponman TJ, Cannon DB. 2000. MNRAS 315:689-702

. Loewenstein M. 1989. MNRAS 238:15-41

. Loewenstein M. 2000. Ap. J. 532:17-27

. Loewenstein M. 2001. Ap. J. 557:573-77 
. Loewenstein M, Mathews WG. 1987. Ap. J. 319:614-31 ADD THIS !

. Loewenstein M, Mathews WG. 1991. Ap. J. 373:445-51

. Loewenstein M, Mushotzky RF. 2002. Ap. J. submitted (astro-ph/0208090)

. Loewenstein M, Mushotzky RF, Angelini L, Arnaud KA, Quataert E. 2001. Ap. J. 555:L21-24

. Loewenstein M, White RE III. 1999. Ap. J. 518:50-63

. Loewenstein M, Zweibel EG, Begelman MC. 1991. Ap. J. 377:392-402

. Macchetto F, Pastoriza M, Caon N, Sparks WB, Giavalisco M, et al. 1996. Astron. Astrophys. Suppl.120:463-488

. Magorrian J, Tremaine S, Richstone D, Bender R, Bower G, et al. 1998. Ap. J. 115:2285-305

. Malagoli A, Rosner R, Fryxell B. 1990. MNRAS 247:367-76

. Martel AR, Sparks WB, Allen MG, Koekemoer AM, Baum SA 2002. Astron. J. 123:1357-63

. Martel AR, Turner NJ, Sparks WB, Baum SA. 2000. Ap. J. Suppl. 130:267-338

. Martin CL, Kobulnicky HA, Heckman TM. 2002. Ap. J. 574:663-92

. Mathews WG. 1989. Astron. J. 97:42-56

. Mathews WG. 1990. Ap. J. 354:468-82

. Mathews WG. 1997. Astron. J. 113:755-63

. Mathews WG, Brighenti F. 1997. Ap. J. Ap. J. 488:595-605

. Mathews WG, Brighenti F. 1998. Ap. J. 493:L9-12

. Mathews WG, Brighenti F. 1999a. Ap. J. 526:114-29

. Mathews WG, Brighenti F. 1999b. Ap. J. 527:L31-34

. Mathews WG, Brighenti F. 2000. Ap. J. 545:181-89

. Mathews WG, Buote DA, Brighenti F. 2001. Ap. J. 550:L31-34

. Matsumoto H, Koyama K, Awaki H, Tomida H, Tsuru, T, et al. 1996. Publ. Astron. Soc. Japan 48:201-10

. Matsushita K. 2001. Ap. J. 547:693-704

. Matsushita K, Belsole E, Finoguenov A, Bohringer H. 2002. Astron. Astrophys. 386:77-96

. Matthias M, Gerhard O. 1999. MNRAS 310:879-91

- McNamara BR. 1997. In In Galactic and Cluster Cooling Flows, ASP Conf. Ser., ed. N. Soker, 115:48-58. San Francisco: Publ. Astron. Soc. Pac.

. McNamara BR. 2001. in Galaxy Clusters and the High Redshift Universe Observed in X-rays, Proc. of XXI Moriond Conference ed. D Neumann, F. Durret \& J. Tran Thanh Van. (in press) (astro-ph/0105563)

. McNamara BR, O'Connell RW, Sarazin CL. 1996. Astron. J. 112:91-104

. McNamara BR, Wise M, Nulsen PEJ, David LP, Sarazin CL, et al. 2000. Ap. J. 534:L135-38

. McWilliam A. 1997. Annu. Rev. Astron. Astrophys. 35:503-56

. Meiksin A. 1988. Ap. J. 334:59-69

. Merluzzi P. 1998. Astron. Astrophys. 338:807-12

. Merritt D, Ferrarese L. 2001. MNRAS 320:L30-34

. Merritt D, Tremblay B. 1996. Astron. J. 111:2243-47

- Molendi S, Gastaldello F. 2001. Astron. Astrophys. 375:L14-17

. Molendi S, Pizzolato F. 2001. Ap. J. 560:194-200

. Morris RG, Fabian AC. 2003. MNRAS 338:824-36

. Moss D, Shukurov A. 1996. MNRAS 279:229-39

. Mulchaey JS. 2000. Annu. Rev. Astron. Astrophys. 38:289-335

. Mushotzky R, Figueroa-Feliciano E, Loewenstein M, Snowden SL, 2003. (astro-ph/0302267)

. Narayan R, Medvedev MV. 2001. Ap. J. 562:L129-32

. Narayan R, Yi I. 1995. Ap. J. 444:231-43

. Navarro JF, Frenk CS, White SD. 1996. Ap. J. 462:563-75

. Nulsen PEJ, David LP, McNamara BR, Jones C, Forman WR, Wise M. 2002. Ap. J. 568:163-73

. Nulsen PEJ, Fabian AC. 2000. MNRAS 311:346-56

- Nulsen PEJ, Stewart GC, Fabian AC. 1984. MNRAS 208:185-95

. Oosterloo TA, Morganti R, Sadler EM, Vergani D, Calwell N. 2002. Astron. J. :123:729-44

. O'Sullivan E. Forbes DA, Ponman TJ. 2001. MNRAS 328:461-84

. Owen FN, Eilek JA, Keel WC. 1990. Ap. J. :449-54

. Pain R, Fabbro S, Sullivan M, Ellis RS, et al. 2002. Ap. J. 577:120-32

. Pellegrini S. 1999. Astron. Astrophys. 351:487-94

- Pellegrini S, Held EV, Ciotti L. 1997. MNRAS 288:1-11

- Peres CB, Fabian AC, Edge AC, Allen SW, Johnstone RM, White DA. 1998. MNRAS 298:416- 
. Peterson JR, Paerels FBS, Kaastra JS, Arnaud M, Reiprich TH, et al. 2001. Astron. Astrophys. 365:L104-109

- Ponman TJ, Cannon DB, Navarro JF. 1999. Nature 397:135-37

- Ponman TJ, Sanderson AJR, Finoguenov A. 2003. MNRAS (in print) (astro-ph/0304048)

. Quilis V, Bower RG, Balogh ML. 2001. MNRAS 328:1091-97

. Rangarajan FVN, Fabian AC, Forman WR, Jones C. 1995. 272:665-77

. Reale F, Rosner R, Malagoli A, Peres G, Serio S. 1991. MNRAS 251:379-90

. Rees MJ, Phinney ES, Begelman MC, Blandford RD. 1982. Nature 252:17-21

. Renzini A. 1997. Ap. J. 488:35-43

. Renzini A. 1999. In Chemical Evolution from Zero to High Redshift ed. J. Walsh \& M. Rosa (Berlin: Springer) 1999, p. 185-93

. Renzini A, Ciotti L. 1993. Ap. J. 416:L49-52

. Rephaeli Y, 1987. MNRAS 225:851-58

. Rephaeli Y, Silk J. 1995. Ap. J. 442:91-95

. Reynolds CS, di Matteo T, Fabian AC, Hwang U, Canizares CR. 1996. MNRAS 283:L111-16

- Reynolds CS, Heinz S, Begelman MC. 2001. Ap. J. 549 L179-82

. Reynolds CS, Heinz S, Begelman MC. 2002. MNRAS 332:271-82

. Rix H-W, de Zeeuw PT, Cretton N, van der Marel RP, Carollo CM. 1997. Ap. J. 488:702-19

. Rizza E, Loken C, Bliton M, Roettiger K, Burns JO, Owen FN. 2000. Astron. J. 119:21-31

. Roberts TP, Warwick RS. 2000. MNRAS 315:98-114

. Rose WK, Guillory J, Beall JH, Kainer S. 1984. Ap. J. 280:550-60

. Rosner R, Tucker WH. 1989. Ap. J. 338:761-69

. Ruszkowski M, Begelman MC. 2002. Ap. J. 581:223-28 (astro-ph/0207471)

. Sadler EM, Oosterloo TA, Morganti R, Karakas A. 2000. Astron. J. 119:1180-96

. Saglia RP, Bertin G, Stiavelli M. 1992. Ap. J. 384:433-47

. Saglia RP, Kronawitter A, Gerhard O, Bender R. 2000. Astron. J. 119:153-61

. Sakelliou I, Peterson JR, Tamura T, Paerels FBS, Kaastra JS et al. 2002 Astron. Astrophys. 391:903-09

. Sarazin CL. 1986. Rev. Mod. Phys. 58:1-116

. Sarazin CL. 1990. In The Interstellar Medium in Galaxies, Proc. of the 2nd Teton Conference, Ed. HA Thronson \& JM Shull (Dordrecht: Kluwer) p. 201-38

. Sarazin CL, Ashe GA. 1989. Ap. J. 345:22-30

. Sarazin CL, Irwin JA, Bregman JN. 2000. Ap. J. 544:L101-105

. Sarazin CL, White RE III. 1987. Ap. J. 320:32-48

. Saxton CJ, Sutherland RS, Bicknell GV. 2001. Ap. J. 563:103-117

- Sazonov SY, Curazov EM, Sunyaev RA. 2002. MNRAS 333:191-201

. Shields JC. 1991. Astron. J. 102:1314-34

. Shigeyama T. 1998. Ap. J. 497:587-93

- Soker N, Sarazin CL. 1990. Ap. J. 348:73-84

- Soker N, Blanton EL, Sarazin CL. 2002. Ap. J. 573:533-41

. Soker N, White RE III, David LP, McNamara BR. 2001. Ap. J. 549:832-39

. Sparks WB. 1992. Ap. J. 399:66-75

. Sparks WB, Macchetto F, Golombek D. 1989. Ap. J. 345:153-62

. Stewart GC, Fabian AC, Nulsen PEJ, Canizares CR. 1984. Ap. J. 278:536-43

- Sulkanen ME, Bregman JN. 2001. Ap. J. :548-L131-34

. Sutherland RS, Dopita MA. 1993. Ap. J. Suppl. 88:253-327

- Tabor G, Binney J. 1993. MNRAS 263:323-34

- Tamura T, Bleeker JAM, Kaastra JS, Ferrigno C, Molendi S. 2001. Astron. Astrophys. 379:10714

. Tawara Y, Akimoto F, Kumada A, Furuzawo A, Yamashita K. 1997. In X-Ray Imaging and Spectroscopy of Cosmic Hot Plasmas, ed. F Makino, K. Mitsuda (Universal Academy Press: Tokyo) p. 87

- Terlevich AI, Forbes, DA. 2002. MNRAS 330:547-62

. Thomas PA. 1986. MNRAS 220:949-69

. Tomita A, Aoki K, Watanabe M, Takata T, Ichikawa S. 2000. Astron. J. 120:123-30

. Toniazzo T, Schindler S. 2001. MNRAS 325:509-30

. Tozzi P, Norman C. 2001. Ap. J. 546:63-84

. Trager SC, Faber SM, Worthey G, González JJ. 2000. Astron. J. 119:1645-76

. Tran HD, Tsvetanov Z, Ford HC, Davies J, Jaffe W, et al. 2001. Astron. J. 121:2928-42 
. Trinchieri G, di Serego Alighieri S. 1991. Astron. J. 101:1647-58

. Trinchieri G, Fabbiano G, Canizares CR. 1986. Ap. J. 310:637-59

. Trinchieri G, Goudfrooij P. 2002. Astron. Astrophys. 386:472-86

- Trinchieri G, Kim D-W, Fabbiano G, Canizares CRC. 1994 Ap. J. 428:555-73

. Trinchieri G, Noris L, di Serego Alighieri S. 1997. Astron. Astrophys. 326:565-79

. Tucker WH, David LP. 1997. Ap. J. 484:602-607

. Tucker WH, Rosner R. 1983. Ap. J. 267:547-50

. van der Marel RP. 1991. MNRAS 253:710-26

. van Dokkum PG, Franx M. 1995. Astron. J. 110:2027-36

. Vader JP. 1986. Ap. J. 305: 669-78

. Vedder PW, Trester JJ, Canizares CR. 1988. Ap. J. 332:725-51

. Vikhlinin A, Markevitch M, Forman W, Jones C. 2001. Ap. J. 555:L87-90

. Voit GM, Donahue M. 1995. Ap. J. 452:164-76

. von Montigny C, Bertsch DL, Chiang J, Dingus BL, Esposito JA, et al. 1995. Ap. J. 440:525-53

. Walsh JR, Walton NA, Jacoby GH, Peletier RF. 1999. Astron. Astrophys. 346:753-63

. White RE III, Chevalier RA. 1983, Ap. J. 275:69-83

. White RE III, Chevalier RA. 1984, Ap. J. 280:561-68

. White RE III, Sarazin CL. 1987. Ap. J. 318:612-20

. White RE III, Sarazin CL. 1991. Ap. J. 367:476-89

. Wiklind T, Combes F, Henkel C. 1995. Astron. Astrophys. 297:643-59

. Xu H, Kahn SM, Peterson JR, Behar E, Paerels FBS, Mushotzky RF, et al. 2002. Ap. J. 579:600-6

. Yoshida T, Habe A, Hattori M. 1991. MNRAS 248:630-41

. Young LM. 2002. Astron. J. 124:788-810

. Zeilinger WW, Pizzella A, Amico P, Bertin G, Bertola F, et al. 1996. Astron. Astrophys. Suppl.120:257-66 